\title{
On the instabilities of a potential vortex with a free surface
}

\author{
Mougel, J.; Fabre, D.; Lacaze, L.; Bohr, Tomas
}

Published in:

Journal of Fluid Mechanics

Link to article, DOI:

10.1017/jfm.2017.341

Publication date:

2017

Document Version

Peer reviewed version

Link back to DTU Orbit

Citation (APA):

Mougel, J., Fabre, D., Lacaze, L., \& Bohr, T. (2017). On the instabilities of a potential vortex with a free surface. Journal of Fluid Mechanics, 824, 230-264. https://doi.org/10.1017/jfm.2017.341

\section{General rights}

Copyright and moral rights for the publications made accessible in the public portal are retained by the authors and/or other copyright owners and it is a condition of accessing publications that users recognise and abide by the legal requirements associated with these rights.

- Users may download and print one copy of any publication from the public portal for the purpose of private study or research.

- You may not further distribute the material or use it for any profit-making activity or commercial gain

- You may freely distribute the URL identifying the publication in the public portal

If you believe that this document breaches copyright please contact us providing details, and we will remove access to the work immediately and investigate your claim. 


\title{
On the instabilities of a potential vortex with a free surface
}

\author{
J. Mougel ${ }^{1,}$, D. Fabre ${ }^{1}$, L. Lacaze ${ }^{1,2}$ and T. Bohr ${ }^{3}$ \\ ${ }^{1}$ Institut de Mécanique des Fluides de Toulouse, Université de Toulouse, Allée du Pr. Camille Soula, \\ 31400 Toulouse, France \\ ${ }^{2}$ CNRS, IMFT, 31400 Toulouse, France \\ ${ }^{3}$ Physics Department and Center for Fluid Dynamics, Technical University of Denmark, \\ 2800 Kongens Lyngby, Denmark
}

In this paper, we address the linear stability analysis of a confined potential vortex with a free surface. This particular flow $\mathrm{h}$ as $\mathrm{b}$ een $\mathrm{r}$ ecently $\mathrm{u}$ sed $\mathrm{b}$ y Toph $\varnothing \mathrm{j}$ et al. (Phys. Rev. Lett., vol. 110(19), 2013, article 194502) as a model for the swirling flow o f fl uid in an op en cy lindrical co ntainer, dr iven by ro tating the bottom plate (the rotating bottom experiment) to explain the so-called rotating polygons instability (Vatistas J. Fluid Mech., vol. 217, 1990, pp. 241-248; Jansson et al., Phys. Rev. Lett., vol. 96, 2006, article 174502) in terms of surface wave interactions leading to resonance. Global linear stability results are complemented by a Wentzel-Kramers-Brillouin-Jeffreys (WKBJ) analysis in the shallow-water limit as well as new experimental observations. It is found that global stability results predict additional resonances that cannot be captured by the simple wave coupling model presented in Tophøj et al. (2013). Both the main resonances (thought to be at the root of the rotating polygons) and these secondary resonances are interpreted in terms of over-reflection $\mathrm{p}$ henomena $\mathrm{b}$ y $\mathrm{t}$ he $\mathrm{W}$ KBJ a nalysis. F inally, w e p rovide experimental evidence for a secondary resonance supporting the numerical and theoretical analysis presented. These different methods and observations allow to support the unstable wave coupling mechanism as the physical process at the origin of the polygonal patterns observed in free-surface rotating flows.

Key words: surface gravity waves, vortex instability, waves in rotating fluids

\section{Introduction}

Rotating free-surface flows are known to offer rich dynamics which can lead to spectacular symmetry breaking of the free surface. A well-known example of such a behaviour can be seen in a simple experiment consisting of a cylindrical container with a rotating bottom, where spontaneous symmetry breaking, leading to rotating polygonal shapes, takes place (Vatistas 1990; Jansson et al. 2006). Recently, Tophøj et al. (2013) have proposed a general mechanism for the emergence of the rotating polygons in terms of surface wave interactions. In their study, the flow in the rotating

$\dagger$ Email address for correspondence: jerome.mougel@imft.fr 
bottom experiment is modelled by an axisymmetric potential vortex (purely azimuthal velocity profile proportional to $1 / r$ ) following experiments and theoretical arguments by Bergmann et al. (2011) and Tophøj \& Bohr (2013). Based on the potential flow assumption, an instability mechanism associated with the coupling of centrifugal modes (near the centre of the cylinder) and gravitational modes (near the lateral walls) was developed into a simple theory for rotating polygons, which we shall refer to as the Tophøj model.

In addition such set-ups can show symmetry breaking of the free surface in the form of switching and sloshing. The switching phenomenon corresponds to a temporal alternation between a polygonal state (only the elliptical case has been reported) and an axisymmetric state (Suzuki, Iima \& Hayase 2006; Iima \& Tasaka 2016). The Tophøj model has recently been shown by Iima \& Tasaka (2016) to be relevant for the rotating bottom experiment in terms of frequencies and mode structure predictions, which confirms that the potential model captures the essence of the instability leading also to the switching phenomenon. In the case of sloshing, as reported by Iga et al. (2014), the free surface also breaks axial symmetry intermittently, but the non-symmetric state has strong deformations close to the outer wall in contrast with the rotating polygons where free-surface deformations are located close to the vortex centre. An explanation of the sloshing phenomenon is proposed by Fabre \& Mougel (2014) using an extension of the Tophøj model to the Rankine vortex.

The Tophøj model only selects specific layers of the flow and thus does not give an accurate description of the shape of the unstable surface. The global stability problem, taking into account the whole free-surface shape, was briefly mentioned in Tophøj et al. (2013) and in Mougel, Fabre \& Lacaze (2014) but not explored in detail. In the present paper, we study the global stability problem presented in $\S 2$ and show that, in addition to the modes taken into account in the Toph $\varnothing \mathrm{j}$ model, modes involving more complex wave structures appear. We show this by giving a detailed report of the instability maps and the associated global mode structures ( $\$ 3)$, a new theoretical interpretation of the instability mechanism which holds for both the rotating polygons and the higher-order shapes by means of Wentzel-Kramers-Brillouin-Jeffreys (WKBJ) analysis in the shallow-water limit $(\S 4)$ and we finally report the first experimental observation of a free-surface polygonal structure with a more complex shape $(\S 5)$.

\section{Global stability approach}

Let us consider a cylindrical tank of radius $R$ partially filled with water. At rest, the free surface is horizontal due to the effect of gravity $g$. The associated filling height is denoted by $H$. We are interested here in instabilities observed in experiments when the bottom plate of the tank is set into rotation (Vatistas 1990; Jansson et al. 2006). As observed by Bergmann et al. (2011), the underlying flow generated by such an apparatus can be well described by a potential vortex in the case of strong rotations of the bottom plate.

\subsection{General equations for free-surface potential flows}

In this section, we derive the general equations governing the motion of an inviscid fluid in the case of a potential flow with a free surface. The problem is solved in a cylindrical coordinate system $(r, \theta, z)$. Denoting by $\Phi$ the velocity potential which is defined by $\boldsymbol{U}=\nabla \Phi$, Laplace's and Bernoulli's equations apply in the fluid volume and read,

$$
\frac{\partial^{2} \Phi}{\partial r^{2}}+\frac{1}{r} \frac{\partial \Phi}{\partial r}+\frac{1}{r^{2}} \frac{\partial^{2} \Phi}{\partial \theta^{2}}+\frac{\partial^{2} \Phi}{\partial z^{2}}=0,
$$



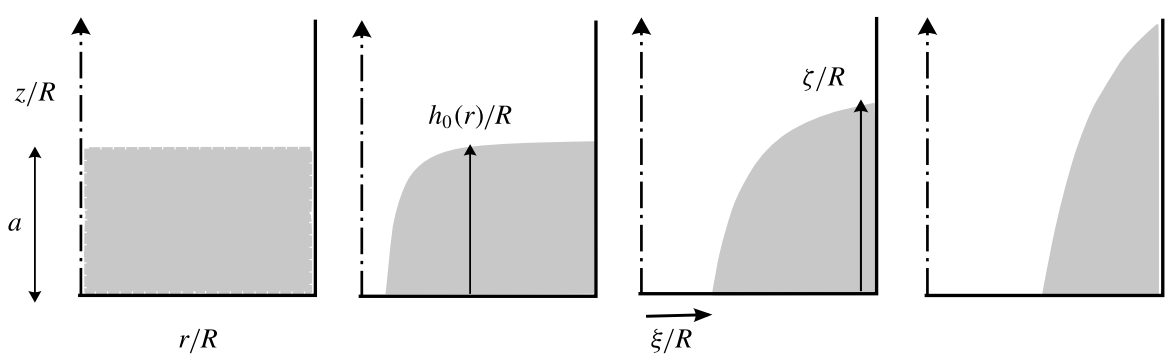

FIgURE 1. Typical free-surface shapes (2.4c) obtained for a given fluid volume and increasing values of $\xi / R$ from left to right: $\xi / R \rightarrow 0, \xi / R=0.1, \xi / R=0.3, \xi / R=0.5$.

$$
\frac{\partial \Phi}{\partial t}+\frac{1}{2} \nabla \Phi \cdot \nabla \Phi+\frac{P}{\rho}+g z=\text { const },
$$

with $P$ the pressure (relative to atmospheric pressure) and $\rho$ the density of the fluid. We now define the free-surface position by the zero level of the implicit function $\mathcal{H}(r, \theta, z, t)=h(r, \theta, t)-z$ with $h$ the vertical height of the free surface and $z=0$ at the bottom of the tank. The kinematic and dynamic boundary conditions on the free surface can therefore be written as

$$
\begin{gathered}
\frac{\partial \mathcal{H}}{\partial t}+\nabla \Phi \cdot \nabla \mathcal{H}=0, \quad \text { at } \mathcal{H}(r, \theta, z, t)=0, \\
P=\gamma \mathcal{C}, \quad \text { at } \mathcal{H}(r, \theta, z, t)=0,
\end{gathered}
$$

with $\gamma$ the surface tension and $\mathcal{C}$ the curvature of the free surface. In the following, the surface tension will be omitted based upon experimental results by Jansson et al. (2006) which indicate that surface tension does not play a crucial role in the formation of the polygonal shape (at least for a set-up with $R \sim 15-20 \mathrm{~cm}$ ).

\subsection{Base flow}

As discussed previously, we suppose that the base flow is entirely azimuthal, corresponding to a steady and axisymmetric potential vortex, for which the velocity field can be written as

$$
U=\Gamma /(2 \pi r) \boldsymbol{e}_{\boldsymbol{\theta}},
$$

where $\Gamma$ is the circulation of the flow. The singularity at $r=0$ induces a strong decrease of the pressure near the centre of the tank which leads to the formation of a dry area close to the axis of symmetry (see figure 1). The velocity potential of the base flow and the associated pressure and free-surface height then take the form

$$
\begin{gathered}
\Phi_{0}(\theta)=\frac{\Gamma}{2 \pi} \theta, \\
\frac{P_{0}(r, z)}{\rho}=\frac{1}{2}\left(\frac{\Gamma}{2 \pi R}\right)^{2}\left(R^{2} / \xi^{2}-R^{2} / r^{2}\right)-g z, \\
h_{0}(r)=\frac{1}{2 g}\left(\frac{\Gamma}{2 \pi R}\right)^{2}\left(\frac{R^{2}}{\xi^{2}}-\frac{R^{2}}{r^{2}}\right),
\end{gathered}
$$

with $\xi$ the size of the inner dry area, meaning that solution (2.4) is only valid for $r>\xi$. For a given volume of fluid, examples of free-surface shapes are shown in figure 1 for 
various values of $\xi / R$, and where $\zeta=h_{0}(R)$. The conservation of the volume between the state at rest and the base flow can be written as $\pi R^{2} H=\int_{\xi}^{R} 2 \pi h_{0}(r) r \mathrm{~d} r$. This leads to the following expressions for $\zeta$ and $\Gamma$

$$
\begin{gathered}
\frac{\zeta}{R}=\frac{H}{R}\left(1-2 \frac{\xi^{2} \ln \frac{R}{\xi}}{R^{2}-\xi^{2}}\right)^{-1}, \\
\frac{\Gamma}{2 \pi \sqrt{g R^{3}}}=\frac{\xi \sqrt{2 H / R}}{\sqrt{R^{2}-\xi^{2}-2 \xi^{2} \ln \frac{R}{\xi}}},
\end{gathered}
$$

parametrized by the normalized radius of the dry area $\xi / R$ and the aspect ratio

$$
a=H / R \text {. }
$$

Note that $\zeta / R$ and $\Gamma /\left(2 \pi \sqrt{g R^{3}}\right)$ are both growing functions of $\xi / R$, hence any of these parameters could be used to characterize the intensity of the vortex. In practice we will present all results in terms of $\xi / R$ as this parameter has a clear geometrical significance, and was already used in Tophøj et al. (2013). Note that for application to the rotating bottom experiment, the dimension of the dry radius $\xi / R$ is controlled by the angular frequency of the rotating bottom. A simple model based on conservation of angular momentum was proposed in Tophøj et al. (2013) and extended in Fabre \& Mougel (2014) to relate these two parameters. We do not develop this argument here, as our goal is not to stick to the specific rotating bottom experiment but to consider the potential vortex model as a more general model which may be relevant to other situations as well.

\subsection{Perturbation equations}

In order to investigate the stability properties of the potential vortex with a free surface, we introduce infinitesimal perturbations of magnitude $\epsilon$ with eigenmode form

$$
\begin{gathered}
\Phi(r, \theta, z, t)=\Phi_{0}(\theta)+\epsilon\left(\phi(r, z) \mathrm{e}^{\mathrm{i}(m \theta-\omega t)}+\text { c.c. }\right), \\
P(r, \theta, z, t)=P_{0}(r, z)+\epsilon\left(\rho p(r, z) \mathrm{e}^{\mathrm{i}(m \theta-\omega t)}+\text { c.c. }\right), \\
h(r, \theta, t)=h_{0}(r)+\epsilon\left(\eta(r) \mathrm{e}^{\mathrm{i}(m \theta-\omega t)}+\text { c.c. }\right) .
\end{gathered}
$$

Note that for the sake of simplicity, the perturbation $p$ has been chosen to have the dimension of a pressure over density to remove $\rho$ in the following which would only appear with the pressure term. Here, $m$ corresponds to the azimuthal wavenumber and $\omega$ is the complex frequency. Any instability of the base flow is then associated with a positive imaginary part of the frequency, denoted in the following $\omega_{i}=\operatorname{Im}(\omega)$. If at least one mode has a positive growth rate for given values of $(a, \xi / R, m)$, the base flow is unstable and a pattern having azimuthal shape $m$ is expected to emerge with growth rate $\omega_{i}$ and rotation frequency $\omega_{r}=\operatorname{Re}(\omega)$.

The perturbation equations are obtained by linearizing the set of equations (2.1) around the base state defined in the previous section, subject to the boundary 
conditions (2.2) together with free slip conditions at the wall of the tank. This leads to the following set of equations

$$
\begin{gathered}
\Delta \phi \equiv\left(\frac{\partial^{2}}{\partial r^{2}}+\frac{1}{r} \frac{\partial}{\partial r}-\frac{m^{2}}{r^{2}}+\frac{\partial^{2}}{\partial z^{2}}\right) \phi=0, \quad \text { in } \mathcal{S}, \\
\mathrm{i}(\omega-m \Omega(r)) \phi=p, \quad \text { in } \mathcal{S}, \\
\mathrm{i}(\omega-m \Omega(r)) \eta=-\sqrt{1+h_{0}^{\prime 2}} \nabla \phi \cdot \boldsymbol{n}, \quad \text { on } \partial \mathcal{S}_{0}, \\
p=g \eta, \quad \text { on } \partial \mathcal{S}_{0}, \\
\nabla \phi \cdot \boldsymbol{n}=0, \quad \text { on } \partial \mathcal{S}_{1} \text { and } \partial \mathcal{S}_{2},
\end{gathered}
$$

with $h_{0}^{\prime}$ the local slope of the unperturbed free-surface shape, which reads $h_{0}^{\prime}=g_{c}(r) / g$, where $g_{c}(r)=\Gamma^{2} /\left(4 \pi^{2} r^{3}\right)$ corresponds to the centrifugal acceleration. Here $\Omega(r)=$ $\Gamma /\left(2 \pi r^{2}\right), \mathcal{S}$ is the resolution domain i.e. $(r, z) \in[\xi, R] \times\left[0, h_{0}(r)\right]$ and $\partial \mathcal{S}$ is its border composed of the mean free surface $\partial \mathcal{S}_{0}$, the bottom wall $\partial \mathcal{S}_{1}$ and the lateral wall $\partial \mathcal{S}_{2}$. In addition, $\boldsymbol{n}$ is defined as the outward normal to the fluid domain $\mathcal{S}$ and the $\nabla$ operator is now defined as $\nabla=[\partial / \partial r, \mathrm{i} m / r, \partial / \partial z]$.

\subsection{Numerical method}

The set of differential equations (2.8) is solved by means of a finite element method. For this purpose, we introduce test functions $\phi^{*}$ and $p^{*}$ associated with $\phi$ and $p$ respectively. The variational formulation of the problem is obtained from $(2.8 a)$ and (2.8b) under the form

$$
\int_{\mathcal{S}}\left[\Delta \phi \phi^{*}-p p^{*}+\mathrm{i}(\omega-m \Omega(r)) \phi p^{*}\right] r \mathrm{~d} r \mathrm{~d} z=0,
$$

and should be valid for any set of test functions $\left[\phi^{*}, p^{*}\right]$. The contribution $\int_{\mathcal{S}} \Delta \phi \phi^{*} r \mathrm{~d} r \mathrm{~d} z$ is then integrated by parts leading to $\int_{\partial \mathcal{S}}[\nabla \phi \cdot n] r \mathrm{~d} s-\int_{\mathcal{S}} \nabla \phi \cdot$ $\bar{\nabla} \phi^{*} r \mathrm{~d} r \mathrm{~d} z$ with $s$ the curvilinear abscissa along the free surface and $\bar{\nabla}$ the complex conjugate of $\nabla$. Border terms are obtained using boundary conditions on $\partial \mathcal{S}_{0}, \partial \mathcal{S}_{1}$ and $\partial \mathcal{S}_{2}$ which are given by $(2.8 c)-(2.8 e)$. From the impermeability condition on the walls, contributions corresponding to $\partial \mathcal{S}_{1}$ and $\partial \mathcal{S}_{2}$ must be zero. Hence, only the border term associated with the free surface is to be retained. Defining two bilinear operators $A$ and $B$ as

$$
\begin{aligned}
A\left(\left[\phi^{*}, p^{*}\right],[\phi, p]\right)= & \int_{\mathcal{S}}\left[\nabla \phi \cdot \bar{\nabla} \phi^{*}+p p^{*}+\mathrm{i} m \Omega(r) \phi p^{*}\right] r \mathrm{~d} r \mathrm{~d} z \\
& -\int_{\partial \mathcal{S}_{0}} \frac{\mathrm{i} m \Omega(r)}{g \sqrt{1+h_{0}^{\prime 2}}} p \phi^{*} r \mathrm{~d} s, \\
B\left(\left[\phi^{*}, p^{*}\right],[\phi, p]\right)= & \mathrm{i} \int_{\mathcal{S}} \phi p^{*} r \mathrm{~d} r \mathrm{~d} z-\mathrm{i} \int_{\partial \mathcal{S}_{0}} \frac{1}{g \sqrt{1+h_{0}^{\prime 2}}} p \phi^{*} r \mathrm{~d} s,
\end{aligned}
$$

the variational formulation can be written as the generalized eigenvalue problem

$$
A\left(\left[\phi^{*}, p^{*}\right],[\phi, p]\right)=\omega B\left(\left[\phi^{*}, p^{*}\right],[\phi, p]\right),
$$


with eigenvector $[\phi, p]$ and corresponding eigenfrequency $\omega=\omega_{r}+\mathrm{i} \omega_{i}$, which should be valid for any set of test functions $\left[\phi^{*}, p^{*}\right]$. It therefore allows us to obtain a dispersion relation in the form $\omega=\mathcal{F}(\xi / R, a, m)$.

Looking at the symmetries of the problem, we see that if $[\phi, p ; \omega]$ is a solution then so is $[\bar{\phi}, \bar{p} ; \bar{\omega}]$, where the bar denotes the complex conjugate. This implies that the eigenvalues will be either pure real (waves) or pairs of complex conjugates (an amplified and a damped mode). This property results from the time-reversal symmetry of the inviscid modelling of the flow used here.

In practice, the problem is discretized by first building a mesh by triangulation of the domain $\mathcal{S}$, and then projecting the unknowns $[\phi, p]$ and the test functions $\left[\phi^{*}, p^{*}\right]$ onto a basis of P1 elements (linear interpolation between the nodes). The resulting matricial eigenvalue problem is eventually solved using a shift-and-invert method. All these operations are performed using the finite element software FreeFEM ++ (see Hecht 2012).

\section{Global stability results}

\subsection{General stability maps}

Figure 2 displays an important result of our work, namely the mapping of the instability regions in the parameter space $(\xi / R, a, m)$ with $2 \leqslant m \leqslant 5$. White areas correspond to parameter regions in which the potential flow is stable and only neutral waves (with real eigenvalues $\omega$ ) are found. Coloured areas correspond to regions where the flow is unstable, with grey levels indicating the corresponding amplification rate. For all values of the azimuthal wavenumber $m$ considered, it is observed that instability occurs in a number of bands. The band with the higher position in the figures, labelled $(0,0)$, is often the one with the largest instability region and highest values of the growth rates. These larger bands will be referred as main resonances in the following. Note that in this paper the term resonance denotes a linear instability resulting from interaction between two waves with the same frequency. In addition to these main resonances, a number of secondary resonances are observed for each value of $m$. The latter consist of thinner bands with lower amplification rates, and are always located at lower values of $\xi / R$ (hence lower values of the vortex intensity $\Gamma)$ than the main ones. In the figures the secondary resonances are labelled with two integers $\left(n_{c}, n_{g}\right)$. As will be explained in the next subsections, these two integers refer to the numbering of the two waves whose interaction is responsible for the resonance.

For wavenumbers $m \geqslant 6$, similar results are obtained, but with instability bands becoming narrower and amplification rates becoming weaker. On the other hand the cases $m=0$ and $m=1$ are found to be stable in the parameter range investigated in figure 2 (see appendix A for a description of the waves existing in these cases).

Figure 3 shows a superposition of all the instability regions obtained for $m=2$ to 4. Considering only the main resonances, the figure shows the same trends as already presented in Tophøj et al. (2013) (see their figure 4b), where instability bands with increasing values of $m$ are successively crossed as the parameter $\xi / R$ (hence the circulation of the vortex) is increased. This trend is also consistent with the fact that, in the rotating bottom experiment, polygonal patterns with an increasing number of corners are successively encountered as the angular velocity of the bottom plate is increased.

Note that in the strictly inviscid case, the number of secondary resonances is theoretically infinite and the corresponding bands get thinner and closer to each 

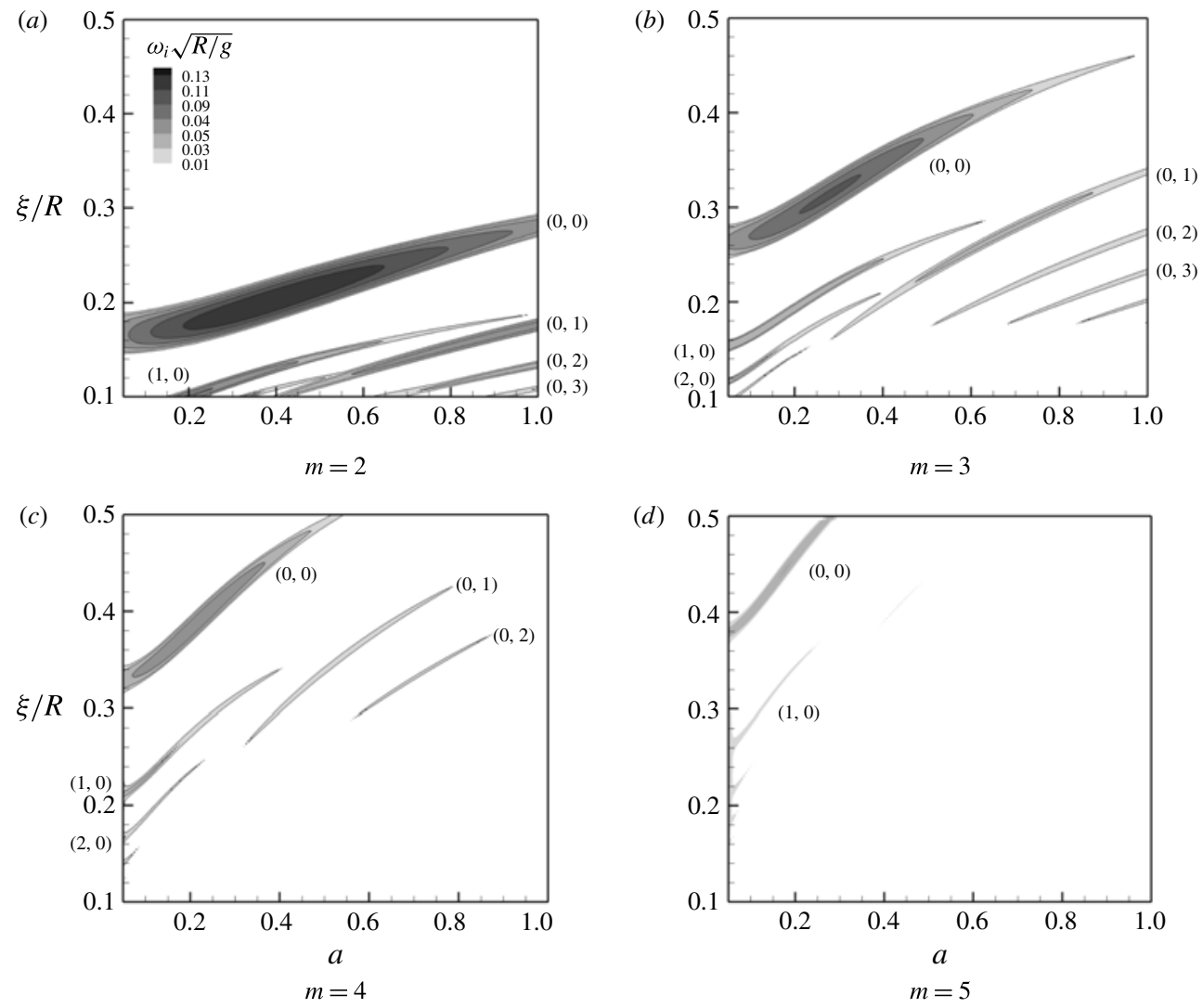

FIGURE 2. Stability maps in the parameter space $(a, \xi / R)$ for different values of $m$. Grey levels correspond to normalized growth rate contours $\left(\omega_{i} \sqrt{g / R}\right)$. A small amount of viscous dissipation is introduced with $C=10^{-4}$ to filter out high-order secondary resonances (see appendix B).

other as $\xi / R$ approaches zero. For visual clarity of the results displayed in figures 2 and 3, an ad hoc procedure was employed to filter out these higher-order resonances. This procedure corresponds to the introduction of a small amount of viscosity. It is explained in appendix B, where it is shown that it has a limited effect on the main resonances and the lowest-order secondary resonances for the value of viscosity considered, while it damps the higher-order, less significant secondary resonances. Note that only figures 2 and 3 (and results shown in appendix B) correspond to potential viscous results, all the other results presented in this paper are purely inviscid.

\subsection{Wave families}

As already stated, the white regions in figure 2 are occupied (in the purely inviscid case) by stable modes with purely real frequencies $\omega$. These modes actually consist of two families of waves whose interaction is at the origin of the instability mechanism. We first document the structure of these waves.

Figure 4 displays the dimensionless frequencies $\omega_{r} \sqrt{R / g}$ of the eigenmodes as a function of the dimensionless radius of the dry area $\xi / R$ for the particular case $a=0.3$ 


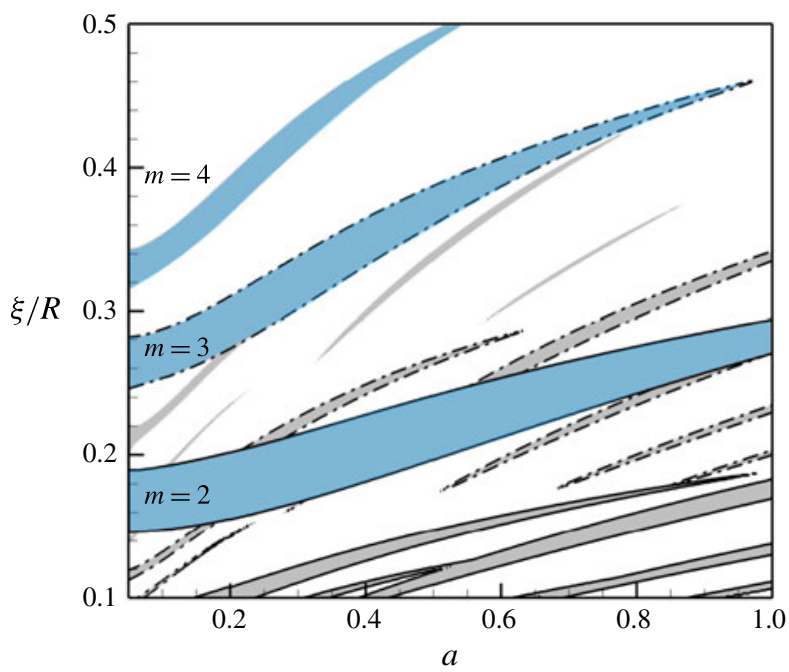

FIGURE 3. (Colour online) Stability map in the parameter space $(a, \xi / R)$ for $m=2$ (plain lines), $m=3$ (dotted lines) and $m=4$ (no lines). Coloured areas correspond to positive growth rate. The main resonances are shown in blue. A small amount of viscous dissipation is introduced with $C=10^{-4}$ to filter out high-order secondary resonances (see appendix B).

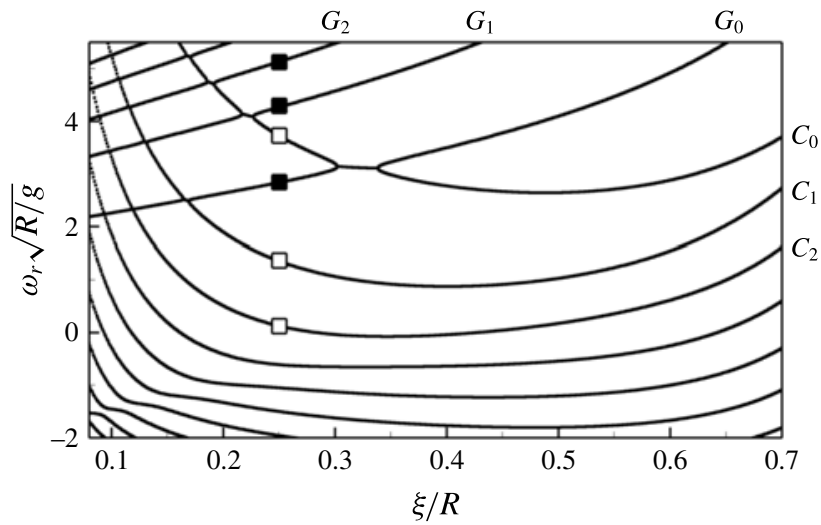

FIGURE 4. Normalized frequencies $\omega_{r} \sqrt{R / g}$ as a function of $\xi / R$ for $a=0.3$ and $m=3$. Squares at $\xi / R=0.25$ correspond to mode structures shown in figure 5 (filled symbols) and figure 6 (empty symbols).

and $m=3$. The frequencies are clearly organized along two sets of branches with different trends.

The first kind of branches are characterized by an increase of the frequency with increasing $\xi / R$. The spatial structure of three different eigenmodes associated with this wave family is shown in figure 5 for the parameters corresponding to the three filled squares in figure 4. As can be seen on the views in a meridional plane, the structure of these modes is mostly concentrated in the region close to the lateral wall of the tank, where the free surface of the base flow is the flattest (see figure 1) as the restoring force acting on the free surface is mostly induced by gravity. These modes are thus 
(a)
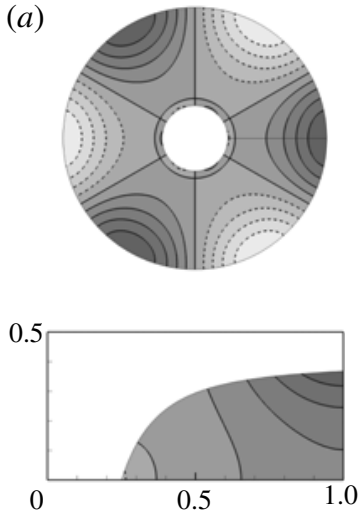

$G_{0}, \omega_{r} \sqrt{R / g}=2.83$ (b)
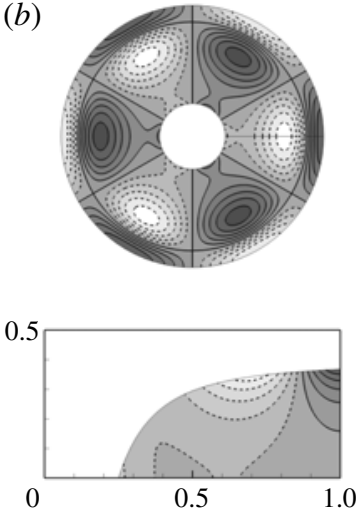

$G_{1}, \omega_{r} \sqrt{R / g}=4.27$ (c)
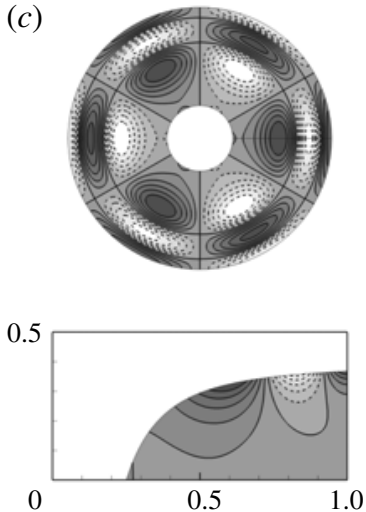

$G_{2}, \omega_{r} \sqrt{R / g}=5.13$

FIGURE 5. Example of gravity modes obtained for $a=0.3, m=3$ and $\xi / R=0.25$ (filled squares in figure 4). Velocity potential contours (real part of $\phi \mathrm{e}^{\mathrm{i}(m \theta-\omega t)}$ ) on the free surface as seen from a top view representation in the $r-\theta$ plane (top row) and in a meridional cross-section in the $r-z$ plane (bottom row) where the meridional cut corresponds to the thin radial line in the corresponding top view figure. Levels are uniformly distributed and dashed lines correspond to negative values. All the displayed structures correspond to neutral waves $\left(\omega_{i} \sqrt{R / g}=0\right)$.

(a)
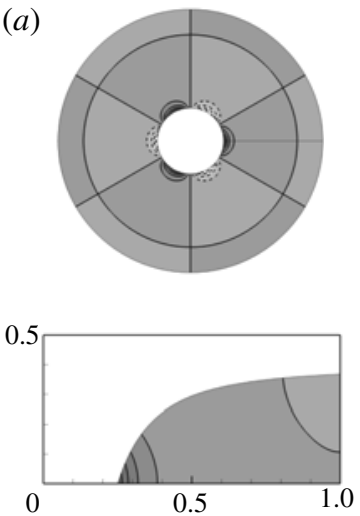

$C_{0}, \omega_{r} \sqrt{R / g}=3.72$ (b)
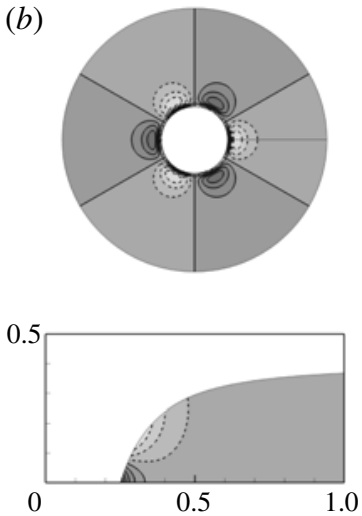

$C_{1}, \omega_{r} \sqrt{R / g}=1.35$ (c)
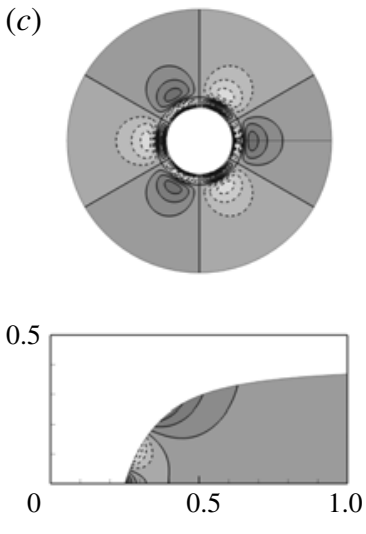

$C_{2}, \omega_{r} \sqrt{R / g}=0.12$

FIgURE 6. Example of centrifugal modes for $a=0.3, m=3$ and $\xi / R=0.25$ (empty squares in figure 4). Contours and conventions are the same as in figure 5 . All the displayed structures correspond to neutral waves $\left(\omega_{i} \sqrt{R / g}=0\right)$.

recognized as gravity waves. Moreover, figure 5 shows that, at a given $\xi / R$, the higher the mode frequency is the more complex its spatial structure is, with in particular an increasing number of nodes in the radial direction. In the following, the corresponding branches will be called $G_{n}$ with $n$ the number of nodes along the free surface in the outer region. $G_{0}$ therefore corresponds to the simplest gravity wave structure without clear nodes (figure $5 a$ ).

The second kind of branches are characterized by a decrease of the frequency with $\xi / R$. The spatial structures of three eigenmodes located along the three first branches 

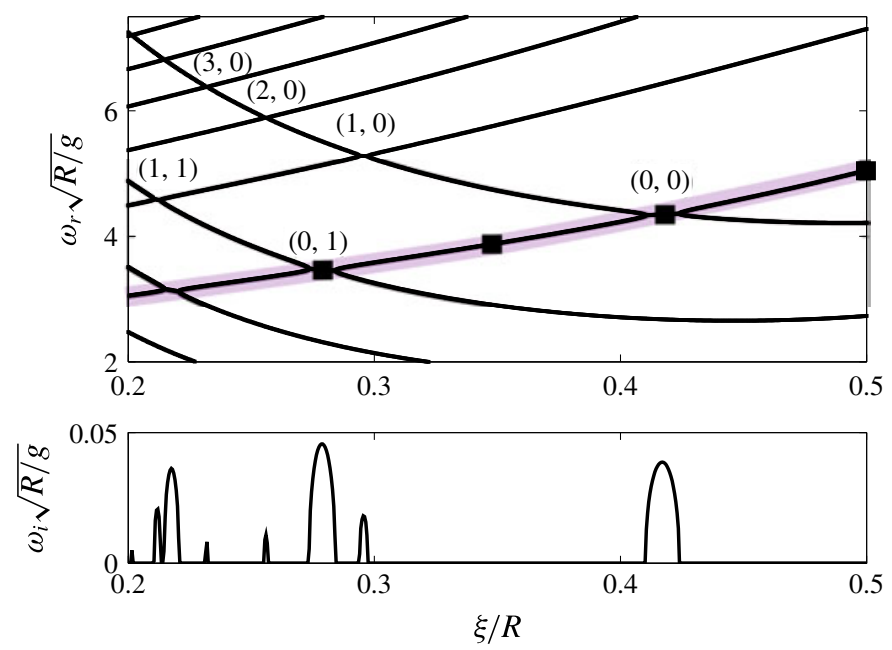

FIGURE 7. (Colour online) Normalized frequencies $\omega_{r} \sqrt{R / g}$ and growth rates $\omega_{i} \sqrt{R / g}$ from global stability as a function of $\xi / R$ for $a=0.7$ and $m=3$. Along $G_{0}$ branch which is highlighted, black squares correspond to mode structures reported in figure 8 .

of this family, for parameters corresponding to the empty squares in figure 4, are displayed in figure 6. These modes have a rather different structure, as they are now localized close to the dry area, where the free surface is almost vertical, i.e. where the restoring force acting on the free surface is mostly induced by the centrifugal acceleration. These modes are thus recognized as centrifugal waves. Similar to the gravity waves, branches are associated with a different mode structure and differ by the number of nodes on the free surface. In this case however, more complex structures are found to correspond to smaller frequencies, a trend which can be understood in the shallow-water limit (see appendix C, equation (C29)). Centrifugal wave branches will be denoted as $C_{n}$ with $n$ the number of nodes along the free surface in the inner region (see figure 6).

\subsection{Wave interaction}

As could be observed in figure 4 , at the locations where branches of gravity and centrifugal waves cross, there is a small interval of $\xi / R$ where one can observe wave frequency merging. In such intervals, the waves which have otherwise real frequencies interact. Such interactions result in the formation of a couple of complex conjugate eigenvalues, and therefore to an instability. In this paragraph, we investigate the details of these interactions and the structure of the resulting eigenmodes.

Let us first consider a rather deep-water case corresponding to $a=0.7$ and $m=$ 3 . Figure 7 displays the frequencies as function of $\xi / R$. In this case, the real parts (upper plot) shows the same trends as already described in figure 4, with two sets of branches interacting as they cross each other. The imaginary parts (lower plot) confirm the existence of an unstable mode in each of the intervals where the waves interact. As already explained these unstable modes are always associated with their stable counterparts with complex conjugate frequencies, but the latter are not considered any longer. Note that in the upper plot, the crossings leading to instabilities are labelled by two indexes $\left(n_{c}, n_{g}\right)$ corresponding respectively to the numbering of the corresponding branches of centrifugal and gravity waves, respectively. 
(a)
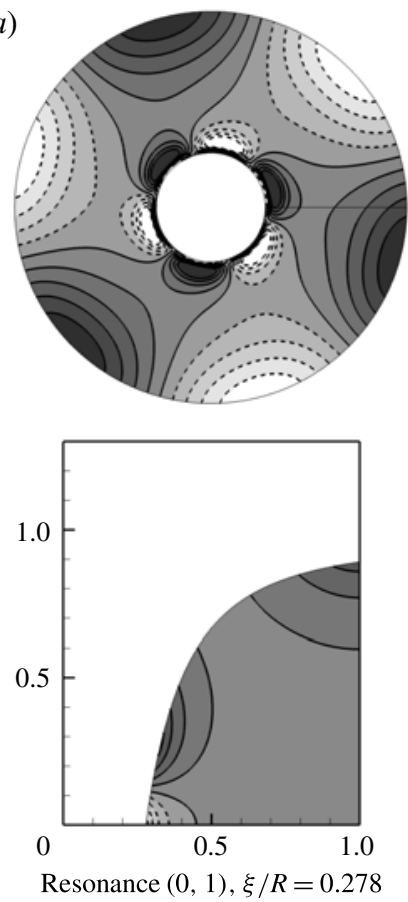

(c)
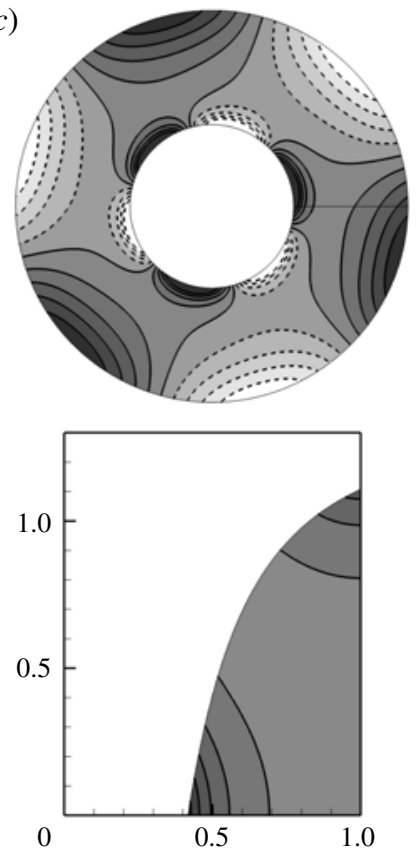

Resonance $(0,0), \xi / R=0.417$ (b)
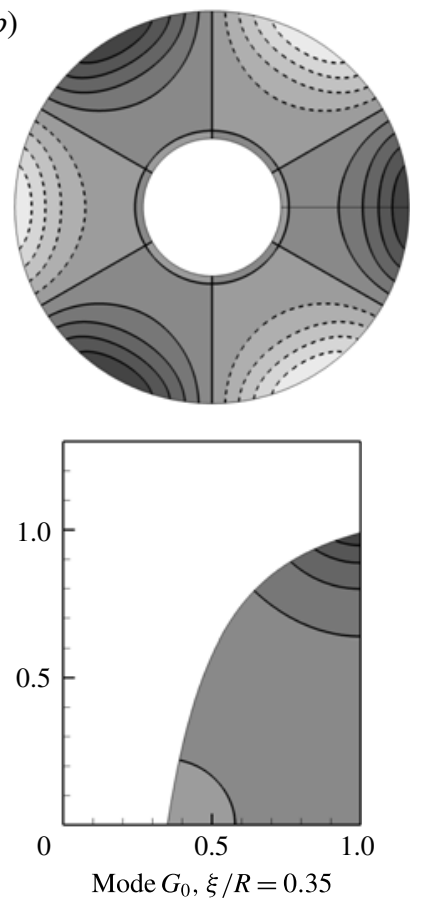

(d)
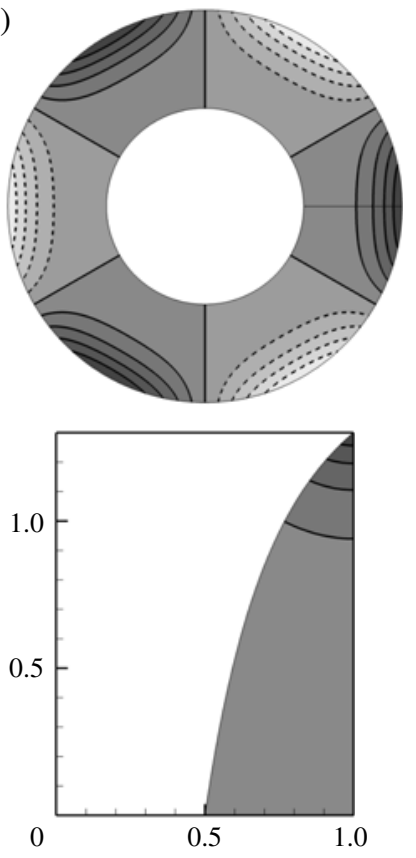

Mode $G_{0}, \xi / R=0.5$

FIgURE 8. Evolution of the structure of $G_{0}$ when $\xi / R$ increases for $a=0.7$ and $m=3$. The corresponding frequency evolution is highlighted in figure 7 and the structures shown here correspond to the black squares on figure 7 . Contours and colour conventions are the same as in figure 5. 
To investigate how the interaction takes place, let us follow the branch associated with the primary gravity wave $G_{0}$, which is highlighted in figure 7 . Figure 8 displays the structure of the eigenmode at four points along this branch, which corresponds to the square symbols in figure 7. Starting from $\xi / R=0.5$ (figure $8 d$ ) is recognized as the pure $G_{0}$ mode as described in $\S 3.2$, with a structure localized near the upper part of the free surface and zero nodes along the free surface. Progressing backwards along this branch, an interaction with the $C_{0}$ branch is observed for $\xi / R \approx 0.417$. At this point the structure of the eigenmode (figure $8 c$ ) clearly shows the presence of both a gravity wave with the same structure as in the previous plot, and of a centrifugal wave localized around the lower part of the free surface, with the characteristic structure of the $C_{0}$ branch already displayed, i.e. without any nodes along the free surface. This instability is the one related to the rotating polygons (here a triangle) according to Tophøj et al. (2013). Note that the gravity wave and centrifugal wave component display a phase shift of a quarter of wavelength in the azimuthal direction, a characteristic of the instability which will be investigated in more detail in the following. Progressing further towards lower values of $\xi / R$, the structure reverts to that of the pure $G_{0}$ wave (figure $8 b$ ) until an interaction with the $C_{1}$ branch arises for $\xi / R \approx 0.278$. At this point, the structure of the eigenmode (figure $8 a$ ) is composed of both the gravity wave $G_{0}$ and the centrifugal wave $C_{1}$ characterized by the existence of one node in the lower part of the free surface.

It is noteworthy that for the rather deep case with $a=0.7$ considered here, the amplification rate associated with the secondary resonance $\left(n_{g}, n_{c}\right)=(0,1)$ is slightly larger than that associated with the main resonance $\left(n_{g}, n_{c}\right)=(0,0)$. This indicates that some secondary resonances may be strong enough to be observed in the experimental set-up for these rather deep-water cases. For $a=0.7$, an experimental evidence of secondary resonance $\left(n_{g}, n_{c}\right)=(0,1)$ corresponding to $m=3$ (triangular pattern) will indeed be discussed in $\S 5$. Note however that for a given value of $m$ and $a$ secondary resonances do not necessarily need to overcome the main instability to be experimentally relevant as they appear for different values of parameter $\xi / R$.

As a second illustration, we now consider a shallow-water case corresponding to $a=0.3$ and illustrate the resonance mechanism for the wavenumber $m=2$. Figure 9 depicts the real and imaginary parts of the frequency as function of $\xi / R$ in the same fashion as in figure 7. In this case, the most powerful secondary resonances occur along the primary branch of centrifugal wave $C_{0}$, which is highlighted in the figure. Figure 10 illustrates the evolution of the eigenmode structure as one moves along this branch. For $\xi / R=0.25$ (figure $10 f$ ) we observe the typical shape of the $C_{0}$ centrifugal mode. When decreasing $\xi / R$ to 0.19 , a first resonance is encountered with the $G_{0}$ mode, leading to the structure illustrated in figure $10(e)$. As in figure $8(c)$, this structure is the superposition of the $G_{0}$ and $C_{0}$ waves, with again a phase shift of a quarter of wavelength in the azimuthal direction between the two components. Moving again downwards along the branch, the structure of the $C_{0}$ wave is recovered (figure $10 d$ ) until a resonance occurs with the $G_{1}$ branch (figure $10 c$ ). The same scenario repeats as $C_{0}$ is recovered again when $\xi / R$ is further increased (figure $10 b$ ) up to a resonance with the $G_{2}$ branch (figure $10 a$ ).

To complete the description of the wave interaction process, figure 11 illustrates the structure of the primary unstable mode for three values of $\xi / R$ corresponding to the lower bound $(\xi / R=0.17)$, centre $(\xi / R=0.19)$ and upper bound $(\xi / R=0.21)$ of the instability interval (as identified by stars in figure 9). This figure allows us to highlight two important features of the wave interaction process. First, as best observed on the evolution of the free-surface displacements at $r=\xi$ and $r=R$ as 


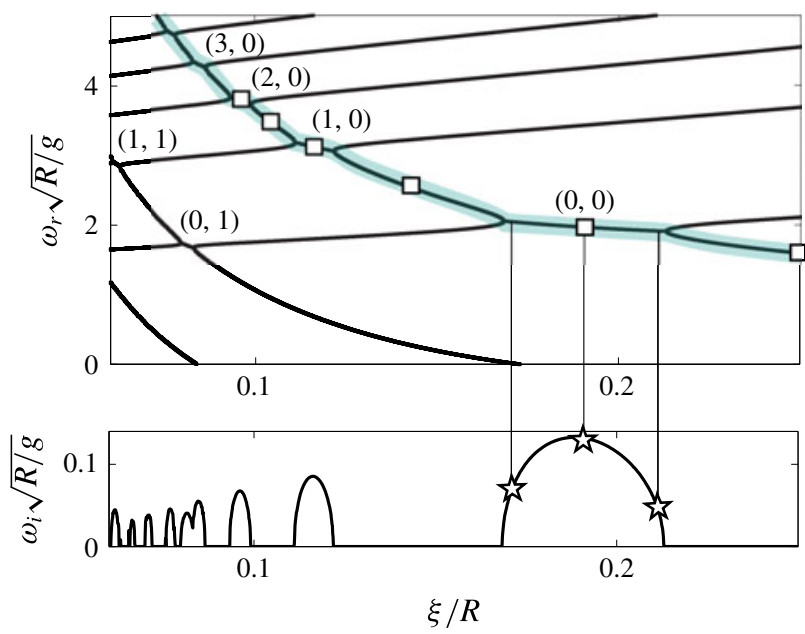

FIgURE 9. (Colour online) Normalized frequencies $\omega_{r} \sqrt{R / g}$ and growth rates $\omega_{i} \sqrt{R / g}$ from global stability as a function of $\xi / R$ for $a=0.3$ and $m=2$. Along $C_{0}$ branch which is highlighted, empty squares (respectively stars), correspond to mode structures reported in figure 10 (respectively figure 11).

function of $\theta$ (lower row), the phase shift $\psi$ (defined in figure 11) between the centrifugal wave and gravity wave components of the mode varies with $\xi / R$. At the lower bound (figure $11 a$ ) the two components are nearly in phase $(\psi \approx 0)$. At the centre of the unstable range corresponding to the maximum amplification, (figure $11 b$ ), both waves are in quadrature $(\psi \approx \pi /(2 m))$ with the gravity wave leading. At the upper bound just before restabilization (figure 11c), the two components end up out of phase $(\psi \approx \pi / m)$. In figure 11 (bottom row), the free-surface displacement at $r=R$ is multiplied by 10 for visual clarity. This gives an order of magnitude of the amplitude ratio between both waves and means that the amplitude of the centrifugal wave is more than ten times larger than that of the gravity wave.

The second important feature illustrated in figure 11 is the existence of a critical radius, defined as the location where the angular velocity of the mode $\omega_{r} / m$ equals that of the base flow $\Omega(r)=\Gamma /\left(2 \pi r^{2}\right)$, i.e.

$$
r_{c}=\sqrt{\frac{m \Gamma}{2 \pi \omega_{r}}} .
$$

This location is displayed by a white circle in the lower plots of figure 11, and is superposed on isocontours of the vertical displacement $\eta$ at the free surface.

The critical radius is found to be located in an intermediate radial region in between radial regions corresponding to gravity and centrifugal waves. In the present case of potential base flow with azimuthal velocity evolving in $1 / r$ (and $z$-independent), the presence of the critical radius in the range $[\xi, R]$ means that the global mode is slower than the base flow at $r=\xi$ and faster at $r=R$.

The fact that instabilities are only possible if gravity and centrifugal waves have nearly the same frequency and have relative velocities with respect to the base flow of opposite sign was already predicted by the Tophøj model which captures 
(a)

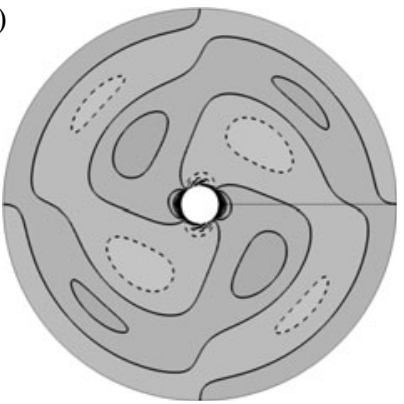

Resonance $(2,0), \xi / R=0.096$

(c)

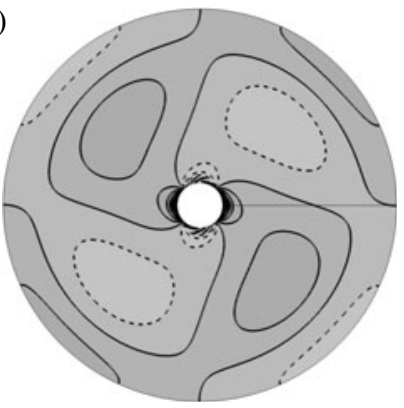

Resonance $(1,0), \xi / R=0.116$

(e)

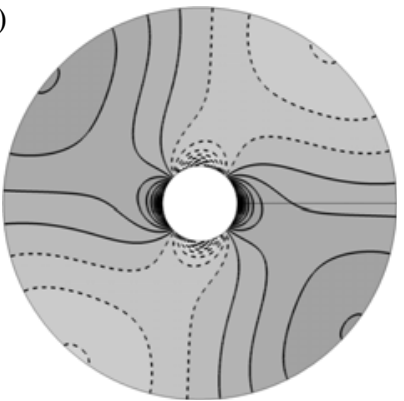

Resonance $(0,0), \xi / R=0.19$ (b)

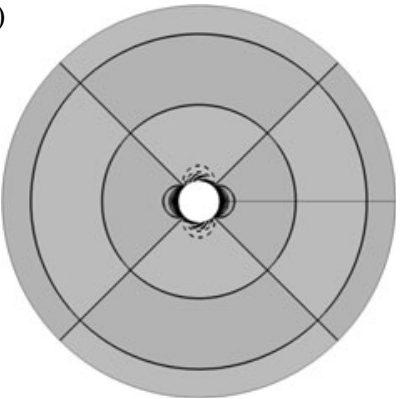

Mode $C_{0}, \xi / R=0.105$

(d)

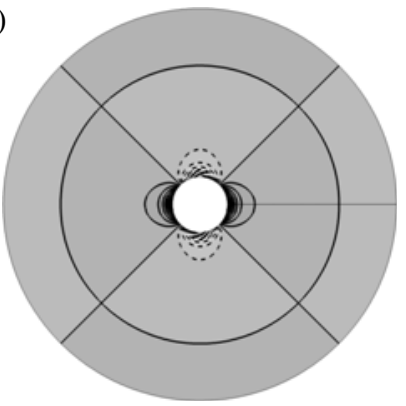

Mode $C_{0}, \xi / R=0.14$

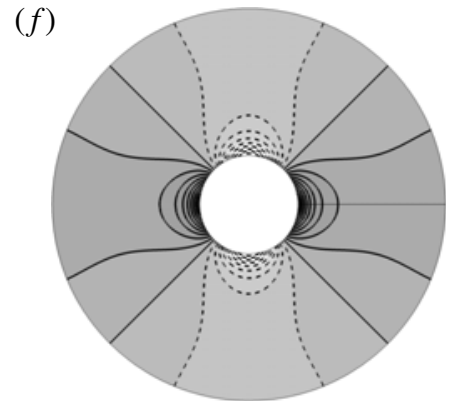

Mode $C_{0}, \xi / R=0.25$

FIGURE 10. Evolution of the structure of $C_{0}$ when $\xi / R$ increases for $a=0.3$ and $m=2$. The corresponding frequency evolution is highlighted in figure 9 and the structures shown here correspond to the black empty squares on figure 9. Only velocity potential contours from the top view representation are shown here. Conventions are identical to figure 5.

only the main resonances. This property actually turns out to be also a necessary condition for secondary resonances. To illustrate this, we display in figure 12 the region in the $\left(\xi-\omega_{r}\right)$ plane for which a critical radius is present in the range $[\xi, R]$ (grey region), superposed on the dispersion relations for the case $a=0.3$ and $m=3$. This figure confirms that all resonances (main and secondary) effectively occur in a range of frequency where a critical radius is present. Note that outside of this range, interactions between two families of waves are also possible but lead to stable near resonances, meaning that the two branches of neutral waves repel and avoid each other instead of merging and giving rise to an unstable mode. This feature is observed in the lower left corner of figure 12 where the irregular behaviour of 
(a)
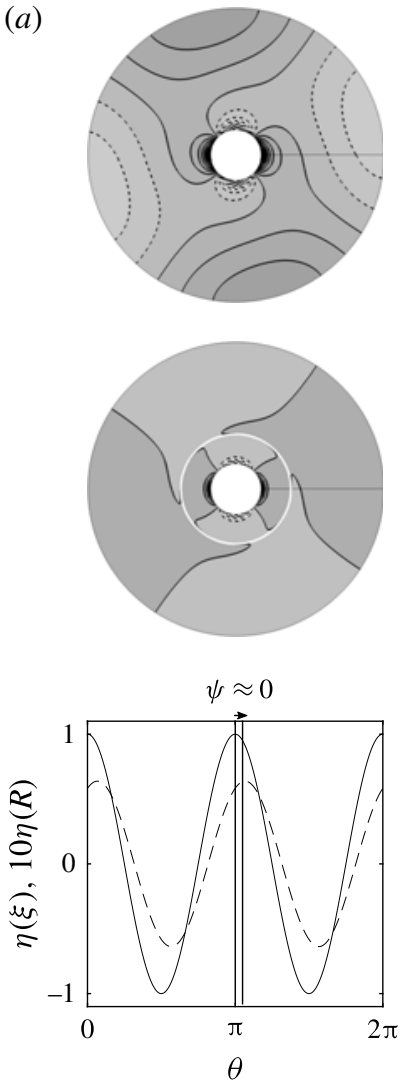

Resonance $(0,0), \xi / R=0.17$ (b)
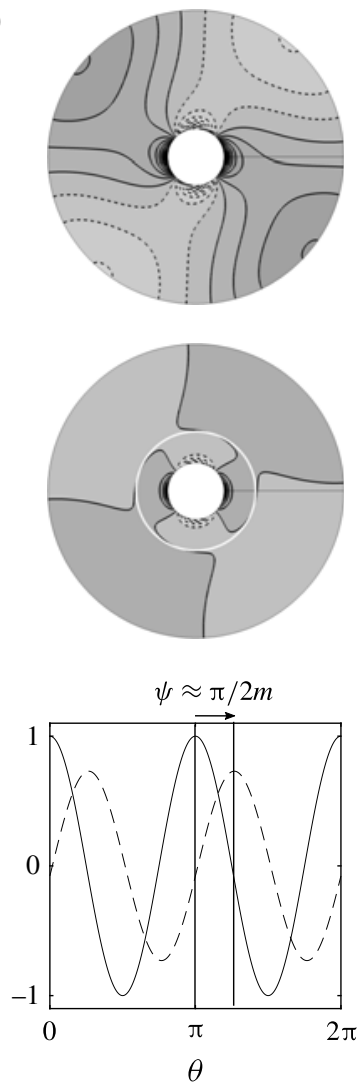

Resonance $(0,0), \xi / R=0.19$ (c)
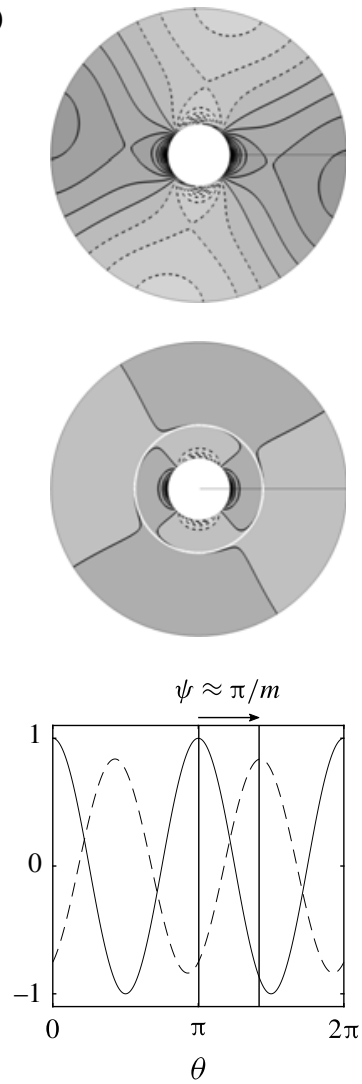

Resonance $(0,0), \xi / R=0.21$

FIGURE 11. Evolution of the structure through the instability $(0,0)$ for $m=2$ and $a=0.3$ (empty stars in figure 9). First row: top view representation for the velocity contours (contours and colour conventions are the same as in figure 5). Second row: free-surface displacement contours (real part of $\eta \mathrm{e}^{\mathrm{i}(m \theta-\omega t)}$ ), white circles indicate the position of the critical radius defined by $r_{c}=\sqrt{m \Gamma /\left(2 \pi \omega_{r}\right)}$. Third row: free-surface displacements at $r=\xi$ (plain line) and $r=R$ (dashed line) as function of $\theta$.

several branches can be explained as a series of near resonances between an almost horizontal gravity wave branch and a number of centrifugal wave branches. Note that these features where already captured by the simple Tophøj model. It is indeed a characteristic feature of instability processes resulting from wave interactions (see. e.g. Cairns (1979)). Such events become more salient in the shallow-water limit presented in the following section.

\section{Asymptotic study in the shallow-water limit}

The instability mechanism is now discussed in the light of the shallow-water limit, an asymptotic expansion allowing a simplification of the linear system. Here it is thus assumed that horizontal scales are much larger than vertical scales, i.e. $a$ is small enough. The aim of this section is to take advantage of the simple shallow-water framework to provide more insight of the instability mechanisms obtained from the global results, namely main and secondary resonances. For that purpose, a WKBJ 


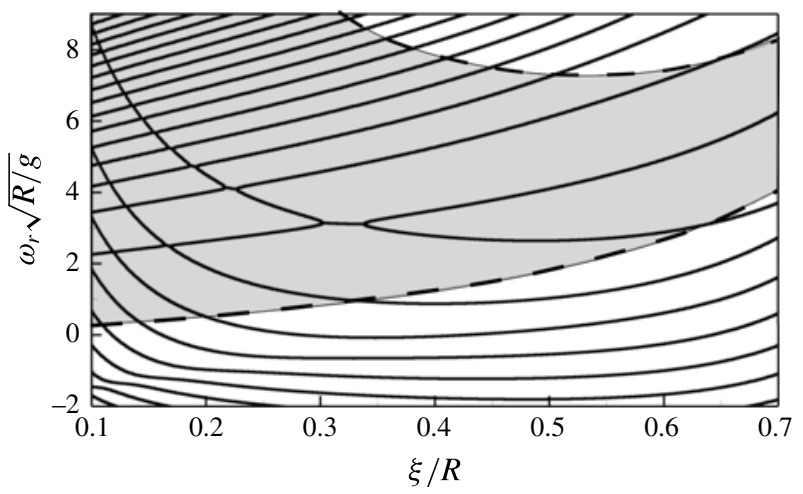

FIGURE 12. Normalized frequencies $\omega_{r} \sqrt{R / g}$ as a function of $\xi / R$ for $a=0.3$ and $m=3$. The shaded area between dashed lines shows the region where a critical radius is present in the fluid domain, i.e. $\xi<r_{c}<R$. Upper dashed line corresponds to $\omega_{r}=m \Omega(\xi)$ (critical radius at $r=\xi$ ), lower dashed line corresponds to $\omega_{r}=m \Omega(R)$ (critical radius at $r=R$ ).

approach is derived in the limit of large $m$, allowing an analytic derivation of approximate dispersion relations thanks to matched asymptotic methods. Details of the WKBJ method are postponed to appendix C.

\subsection{Shallow-water approximation}

In the shallow-water approximation, the flow is supposed to be vertically confined and the flow properties are weakly dependent upon the vertical coordinate. Thus, we can infer an expression for the potential flow with the form

$$
\phi(r, z) \approx \hat{\phi}(r)\left(1+\frac{K(r)}{2} z^{2}\right) .
$$

If $K(r)$ is an order-one function, since $z$ is assumed small with respect to the other dimensions of the problem, the last term in (4.1) is effectively a small correction to an otherwise two-dimensional flow. This ansatz also automatically satisfies the boundary condition $(2.8 e)$ at the bottom. Inserting this expression into (2.8a) leads at leading order to

$$
\Delta_{2} \hat{\phi}(r)+K(r) \hat{\phi}(r)=0,
$$

where $\Delta_{2} \equiv\left(\partial^{2} / \partial r^{2}+1 / r \partial / \partial r-m^{2} / r^{2}\right)$ is the two-dimensional Laplacian operator. Recognizing that $\sqrt{1+h_{0}^{\prime 2}} \nabla \phi \cdot \boldsymbol{n}=\partial \phi / \partial z-h_{0}^{\prime} \partial \phi / \partial r$, where $h_{0}(r)$ is the unperturbed free surface given by $(2.4 c)$, and using (4.1), $(2.8 d)$ and $(2.8 b)$, the kinematic boundary condition $(2.8 c)$ leads at leading order to

$$
h_{0} K(r) \hat{\phi}(r)=h_{0}^{\prime} \frac{\mathrm{d} \hat{\phi}}{\mathrm{d} r}+\frac{(\omega-m \Omega(r))^{2}}{g} \hat{\phi}(r) .
$$

Inserting this latter expression into (4.2) eventually leads to a single ordinary differential equation of the form

$$
\frac{\mathrm{d}^{2} \hat{\phi}}{\mathrm{d} r^{2}}+\left(\frac{1}{r}+\frac{h_{0}^{\prime}}{h_{0}}\right) \frac{\mathrm{d} \hat{\phi}}{\mathrm{d} r}-m^{2} \Lambda(r) \hat{\phi}=0,
$$




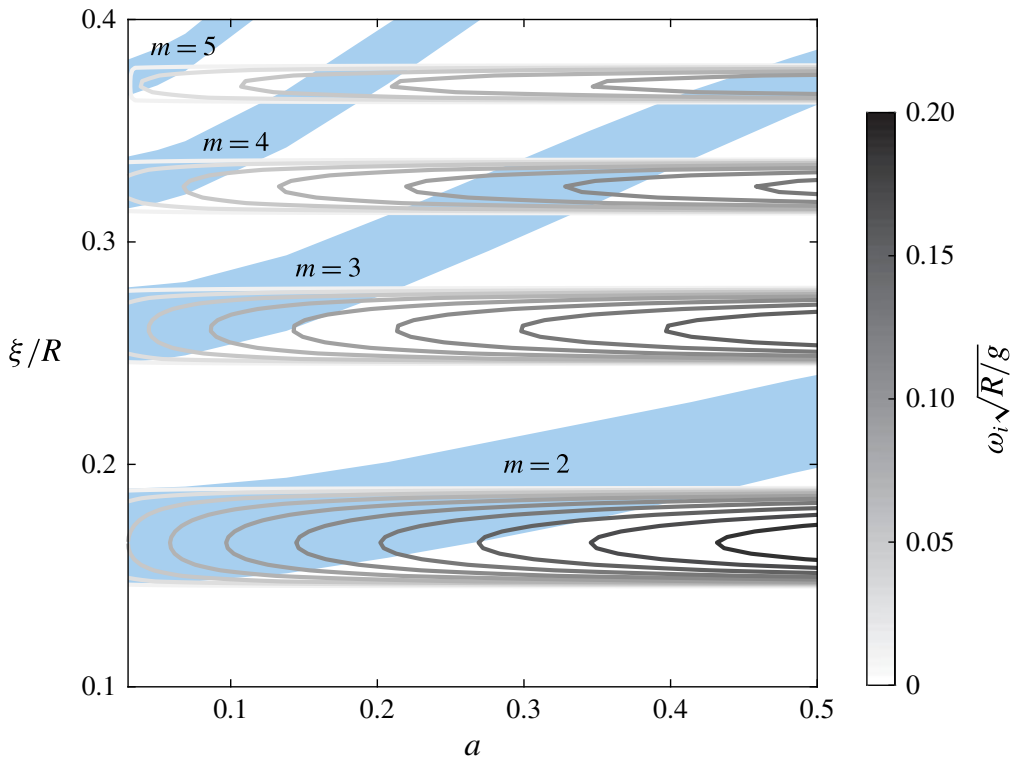

FIGURE 13. (Colour online) Instability map for the main resonance $(0,0)$ and for $m=2,3,4$ and 5. Comparison between the numerical solution of the shallow-water equation, (4.4), (contours of normalized growth rate are shown in grey scales) and the instability map obtained from the global stability approach detailed in $\S 2$ (blue zones).

with

$$
h_{0}(r)=\frac{1}{2 g}\left(\frac{\Gamma}{2 \pi R}\right)^{2}\left(\frac{R^{2}}{\xi^{2}}-\frac{R^{2}}{r^{2}}\right),
$$

and

$$
\Lambda(r)=\frac{1}{r^{2}}-\frac{1}{g h_{0}}\left(\frac{\omega}{m}-\Omega(r)\right)^{2} .
$$

The shallow-water equation, (4.4), can be solved numerically by means of a shooting method and the instability map in the parameter space $(a, \xi / R)$ is shown in figure 13 for $m$ ranging from 2 to 5. Here, only the main unstable interactions are considered and compared to the global stability results. As can be observed in figure 13, the shallow-water model captures the main features of the instability map. In particular, the instability appears as successive bands in the parameter space with an important influence of $\xi / R$. While the global stability highlights a clear dependence of $a$ on unstable regions, the shallow-water approximation only shows a dependence of the growth rate of the instability. However, the obtained unstable regions and growth rates predicted by the shallow-water model perfectly match the global stability results for small $a$ (see figure 13). The shallow-water limit therefore appears as a good candidate to highlight some features of the instability.

\subsection{WKBJ approximation for the shallow-water equation}

The fact that the last term of the shallow-water equation, (4.4), is proportional to $m^{2}$ makes it well suited for a WKBJ approach for large $m$ as explained in the 


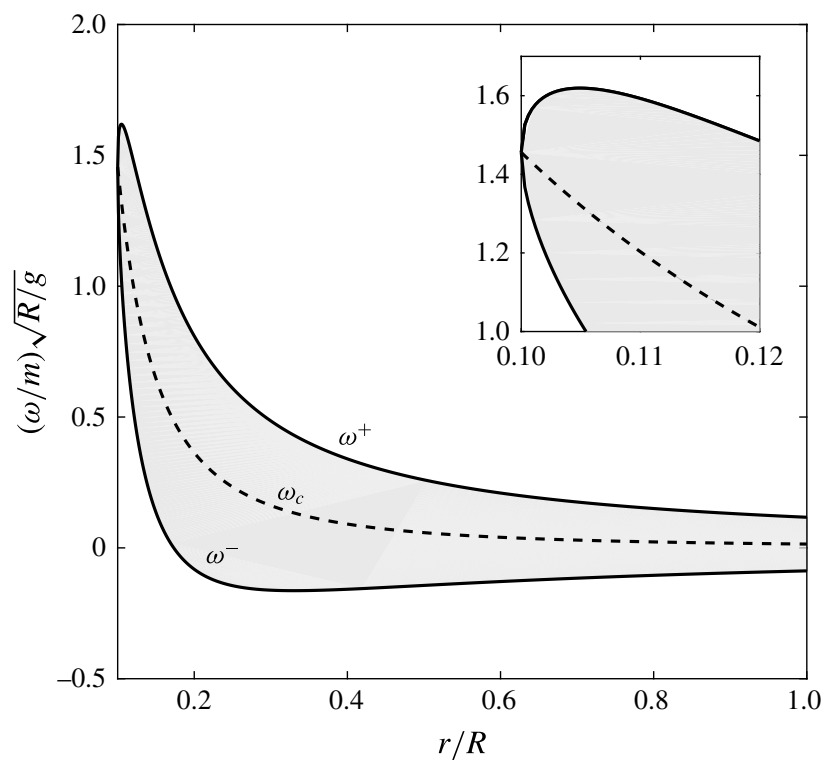

FIGURE 14. Epicyclic frequencies obtained for $a=0.01$ and $\xi / R=0.1$. Thick lines correspond to the normalized epicyclic frequencies $\left(\omega^{ \pm} / m\right) \sqrt{R / g}$ given by (4.7), dashed line corresponds to the normalized critical frequency $\left(\omega_{c} / m\right) \sqrt{R / g}$. Grey area corresponds to $\Lambda>0$ (evanescent behaviour), white areas to $\Lambda<0$ (oscillatory behaviour). The inset shows a magnification near $r=\xi$.

appendix C. This kind of large $m$ WKBJ approach has already been used by Ford (1994) to study other velocity profiles of a shallow-water vortex with a free surface. The WKBJ approach allows us to discuss the nature of the solutions of (4.4) based upon the sign of $\Lambda$. For this discussion, $\omega$ is assumed to be real.

For a given $\omega / m$, radial positions where $\Lambda(\omega / m, r)<0$ correspond to oscillatory solutions while solutions are evanescent where $\Lambda(\omega / m, r)>0$. This leads us to introduce the epicyclic frequencies $\omega^{ \pm}(r)$ (Le Dizès \& Lacaze 2005) defined such as $\Lambda\left(\omega^{ \pm} / m, r\right)=0$ for $r \in[\xi, R]$, along which the WKBJ approximation breaks down. From (4.6), one can obtain

$$
\frac{\omega^{ \pm}(r)}{m}=\Omega(r)\left(1 \pm \sqrt{\frac{1}{2}\left(\frac{r^{2}}{\xi^{2}}-1\right)}\right) .
$$

Conversely, for a given $\omega / m$, radial solutions corresponding to $\Lambda(\omega / m, r)=0$ are the so-called turning points $r_{t i}$ (with $i=1$ or 2 in the present case) of the WKBJ approximation. Moreover, one can define a critical frequency $\omega_{c}(r)=m \Omega(r)$ for which the associated mode exhibits a critical radius at location $r$. For a given solution, the critical radius $r_{c}=\sqrt{m \Gamma /(2 \pi \omega)}$ delineates two radial regions where waves travel faster and slower than the base flow as mentioned in the previous section.

Figure 14 shows the epicyclic frequencies together with the critical frequency as a function of $r / R$ for $a=0.01$ and $\xi / R=0.1$. In this figure, oscillatory and evanescent behaviours of the solution correspond to white and grey zones respectively. Note that the $\omega_{c}$ curve lies in the evanescent region. Depending on the value of $\omega / \mathrm{m}$ for the mode considered, different radial configurations can be anticipated. In particular, 


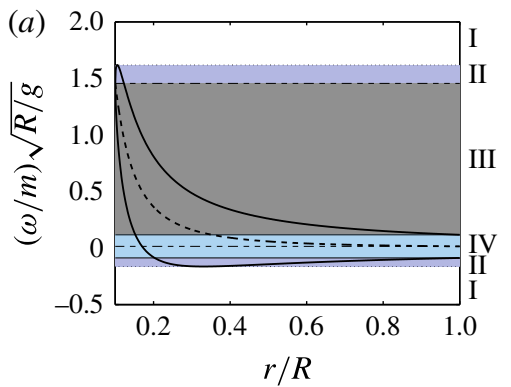

(b)

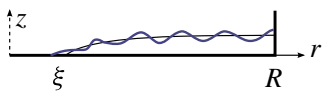

Config. I

(d)

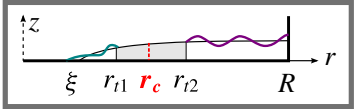

Config. III (c)

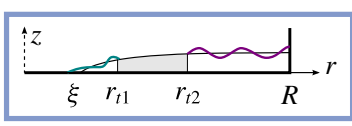

Config. II

(e)

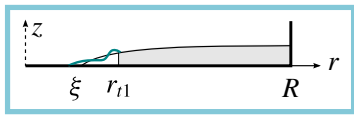

Config. IV

FIGURE 15. (Colour online) (a) Epicyclic frequencies obtained for $a=0.01$ and $\xi / R=0.1$ (same as figure 14) where the different possible configurations depending upon the frequency values are shown in colours. The horizontal lines from top to bottom respectively correspond to $\max \left(\omega^{+}(r)\right)$ (dotted), $\omega_{c}(\xi)$ (dashed), $\omega^{+}(R)$ (plain), $\omega_{c}(R)$ (dashed), $\omega^{-}(R)$ (plain) and $\min \left(\omega^{-}(r)\right)$ (dotted). $(b-e)$ Sketches of the obtained configurations.

a horizontal line in figure $15(a)$, which corresponds to a given solution $\omega / m$, may cross (or not) epicyclic and critical frequency curves. This would indicate a change in the behaviour of the solution (oscillatory versus evanescent) and a change in relative velocity sign between the wave and the base flow respectively. Different configurations of radial structures are obtained here, which are highlighted in figure 15 $(a)$ where each configuration is identified by a colour (configurations I, II, III, IV). A sketch of the radial structure of the solution for the different configurations (configurations I, II, III, IV) is shown in figure $15(b-e)$. These different configurations are now described in more details.

(i) Configuration I: $\Lambda<0$ for all radial positions and no critical radius. The spatial structure of the associated mode corresponds to the one shown in 15(b). For this purely oscillatory configuration, we do not expect to find two well-separated wave families.

(ii) Configuration II: $\Lambda$ changes sign twice along the radial direction and no critical radius. In this case, two oscillatory zones are present and separated by an evanescent zone. This corresponds to figure $15(\mathrm{c})$. The oscillatory area in the neighbourhood of $\xi$ is associated with centrifugal waves while the oscillatory area close to the external cylinder corresponds to gravity waves. As will be seen these two wave families can be clearly distinguished due to the existence of the evanescent spacial separation.

(iii) Configuration III: $\Lambda$ changes sign twice along the radial direction and a critical radius is present. In this case, two oscillatory zones are present and separated by an evanescent zone which now includes a critical radius (figure 15d). This configuration will be of main importance in the following discussion.

(iv) Configuration IV: $\Lambda$ changes sign once along the radial direction and a critical radius can be present. In this case, an oscillatory zone is present close to the inner contact line, while the area enclosing the external cylinder is evanescent (figure 15e). Note that this configuration could include or not a critical radius. The distinction has not been performed in this paper for simplicity.

The location of these different configurations are now reported in figure 16 in which non-dimensional frequencies are plotted as a function of $\xi / R$ in the case 


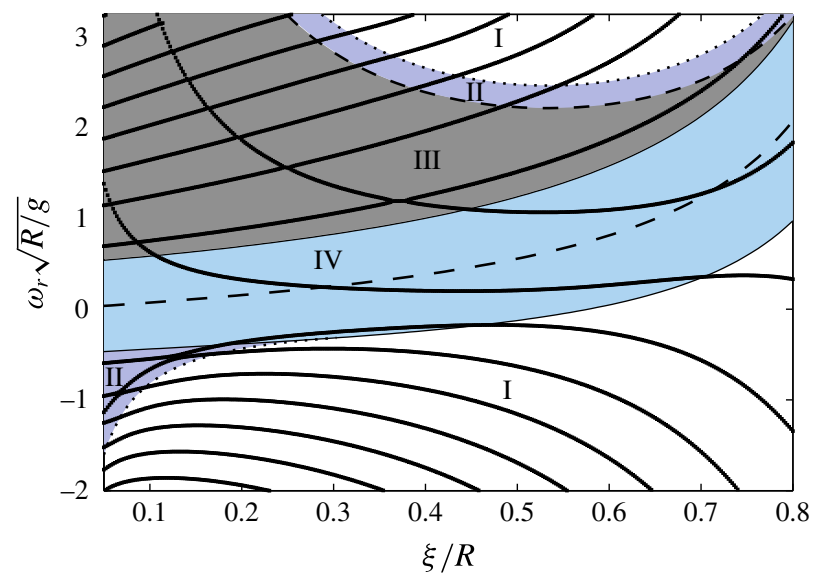

FIgURE 16. (Colour online) Normalized frequencies $\omega_{r} \sqrt{R / g}$ as function of $\xi / R$ for $m=5$ and $a=0.01$. Superposition of global stability results (black thick lines) and areas corresponding to the different configurations presented in figure 15. Legends and colours conventions are identical to figure 15.

$m=5$ and $a=0.01$. These values of $a$ and $m$ have been chosen to satisfy both the shallow-water approximation and the WKBJ approximation, respectively. In figure 16, black lines correspond to the solutions obtained with the linear stability analysis, and the different zones discussed previously from the WKBJ analysis are delimited thanks to $\omega^{ \pm}(R)$, $\min \left(\omega^{-}(r)\right)$, $\max \left(\omega^{+}(r)\right)$ and $\omega_{c}(\xi)$. Eigenmodes exhibit a critical radius if their dimensionless frequency lies in the interval $\left[\omega_{c}(R), \omega_{c}(\xi)\right]$, a region delimited by the dashed lines in figure 16. The general picture of the frequency branches from the global stability in this shallow case $(a=0.01)$ is found to be qualitatively similar to the one discussed in $\S 3(a=0.3)$. In particular, we recover gravity and centrifugal wave branches, as well as resonances and near resonances. It has been verified in figure 16 that wave crossings in region III indeed correspond to resonance leading to instability (at $\xi / R \approx 0.37, \omega \sqrt{R / g} \approx 1.2$ for instance), while features where branches seem to cross in region II correspond to near resonances without instability (at $\xi / R \approx 0.14, \omega \sqrt{R / g} \approx-0.5$ for instance). It is worth mentioning that the regions were gravity waves and centrifugal waves coexist and are clearly distinguished, correspond to region II and region III (see figure 16). This is in accordance with the spatial structure obtained from the WKBJ analysis and shown in figure $15(c, d)$. In region IV, only centrifugal branches are found in agreement with the WKBJ structure shown in figure 15(e). In addition, it can be seen in figure 16 that wave families (gravity and centrifugal) do not coexist in region I.

Once focusing only on regions II and III, it is interesting to note that the nature of the wave interaction, leading to a resonance or a near resonance, respectively, depends on the presence or not of a critical radius in the evanescent region of the mode. We therefore recover the importance of the critical radius introduced in the previous section (see figure 12 for instance).

We conclude that the predicted WKBJ structures are strongly correlated to the global stability results and more precisely to the possible occurrence of instability.

In order to investigate the instability mechanisms in more depth, we now focus on configuration III (corresponding to two wavy regions separated by an evanescent region which includes a critical radius), and use asymptotic matching techniques 

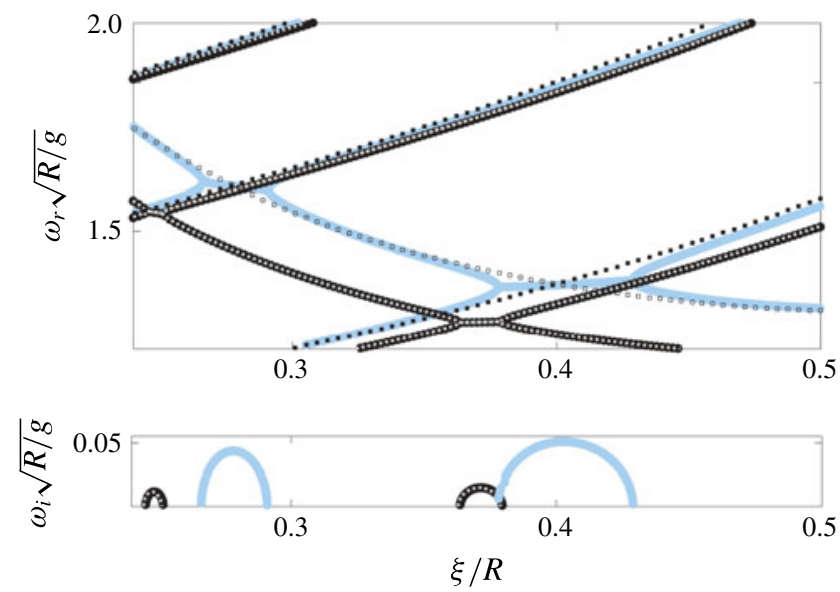

FIgURE 17. (Colour online) Normalized frequencies $\omega_{r} \sqrt{R / g}$ and growth rate $\omega_{i} \sqrt{R / g}$ in the case $m=5$ and $a=0.01$. Comparison between global stability (thick black lines), numerical solution of the shallow-water equation (thin dotted line approximately on top of the global results), WKBJ results (thick blue line) and the simplified dispersion relations for the first centrifugal wave: $\omega=m \Omega(\xi)-\sqrt{m g_{c} / \xi}$ (open squares) and gravity waves: $m W_{2 R}(\omega)=\pi / 4+n_{g} \pi$ with $n_{g}=0,1,2$ (filled squares).

for $m \gg 1$ to obtain approximate dispersion relations. Technical details associated with the WKBJ method are reported in appendix $\mathrm{C}$ and the obtained dispersion relation corresponds to (C29). Only the case corresponding to the first centrifugal wave (e.g. $\left.n_{c}=0\right)$ is discussed here. In this case (C 29) leads to

$$
D_{c}(\omega) D_{g}(\omega)=\sqrt{g_{c}(\xi) \frac{m}{\xi}} \mathrm{e}^{-2 m W_{12}(\omega)}
$$

with

$$
\begin{aligned}
& D_{g}(\omega)=1 / \tan \left(m W_{2 R}(\omega)+\pi / 4\right), \\
& D_{c}(\omega)=\omega-m \Omega(\xi)+\sqrt{g_{c}(\xi) \frac{m}{\xi}}
\end{aligned}
$$

and

$$
W_{2 R}(\omega)=\int_{r_{t 2}}^{R} \sqrt{-\Lambda(r)} \mathrm{d} r \quad \text { and } \quad W_{12}(\omega)=\int_{r_{t 1}}^{r_{t 2}} \sqrt{\Lambda(r)} \mathrm{d} r
$$

where $W_{12}(\omega)$ characterizes the potential barrier separating the oscillatory regions delimited by the two turning points $r_{t 1}$ and $r_{t 2}$, and $W_{2 R}(\omega)$ corresponds to the external oscillatory region associated with gravity waves.

Solutions of (4.8) are compared to the numerical solutions of (4.4) and global stability results for $a=0.01$ and $m=5$ in figure 17. From this figure, it can be seen that the main features of the instability are well captured by the WKBJ approach although the position of unstable resonances and associated growth rates are not perfectly reproduced. In addition, figure 17 also shows the solutions corresponding 

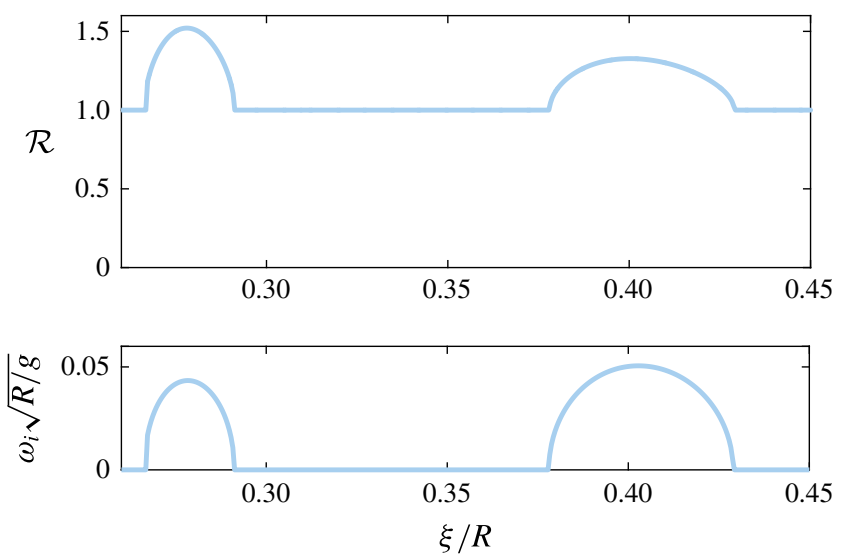

FIGURE 18. (Colour online) Reflection coefficient $\mathcal{R}$ and normalized growth rate $\omega_{i} \sqrt{R / g}$ as function of $\xi / R$ for $m=5$ and $a=0.01$. WKBJ results.

to $D_{c}(\omega)=0$ and $D_{g}(\omega)=0$, which fall on top of the solutions of the complete dispersion relation (4.8) except close to resonances, and are recognized to correspond to centrifugal and gravity waves respectively. Dispersion relation (4.8) therefore describes two wave families which can interact if there natural frequencies are close. The intensity of the coupling is found to be proportional to $\mathrm{e}^{-2 m W_{12}(\omega)}$ and vanishes if the evanescent region is large or for large $m$. This latter trend is in qualitative agreement with results shown in figures 3 and 13 where both the growth rate and the size of the unstable bands are found to decrease with $m$.

In addition, the WKBJ theory allows us to compute the reflection coefficient of a wave at a turning point $r_{t i}$, delimiting an oscillatory solution with an evanescent region (see Billant \& Le Dizès 2009; Park \& Billant 2013, for instance). In the present case for configuration III, the reflection coefficient of the gravity wave at $r_{t 2}$ can be obtained and is shown in appendix $\mathrm{C}$ to read

$$
\mathcal{R}=\left|\exp \left(\mathrm{i} 4 m W_{2 R}(\omega)\right)\right| .
$$

This expression shows that $\mathcal{R}=1$ if $\omega$ is purely real, but may be different from one otherwise. This can be seen in figure 18, which shows that the reflection coefficient is unity where $\omega_{i}=0$, and larger than unity for $\omega_{i}>0$, i.e. for solutions corresponding to either main or secondary unstable resonances. The instability mechanism for both main and secondary resonances is therefore associated with wave over-reflection. This process has already been described in several cases (see Acheson 1976; Lindzen \& Barker 1985; Takehiro \& Hayashi 1992; Billant \& Le Dizès 2009; Park \& Billant 2013 , for instance). The role of the critical radius on the reflection mechanism has been highlighted in these studies and is also found to be crucial in the present case. In addition the link between over-reflected waves and instability is discussed by Takehiro \& Hayashi (1992) and more recently by Billant \& Le Dizès (2009). It requires configuration of type III, i.e. two wavy regions separated by an evanescent area, as well as an additional boundary (the wall at $r=R$ or the contact line at $r=\xi$ in our case) to reflect back the over-reflected wave and therefore allowing for multiple over-reflections.

This point of view of the instability mechanism in terms of over-reflection comes as a complement of the interpretation of Tophøj et al. (2013) in terms of wave 
(a)

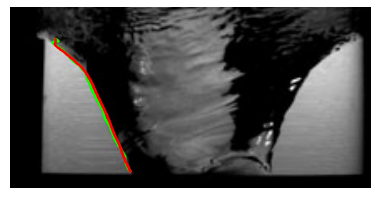

$t=0$

(c)

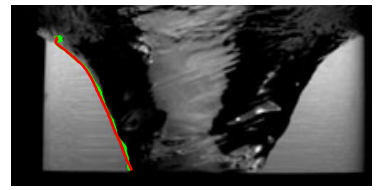

$t=T / 2$

(e)

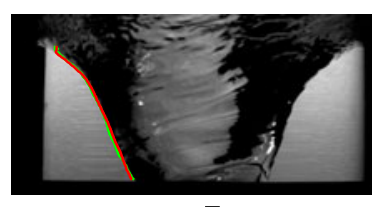

(b)

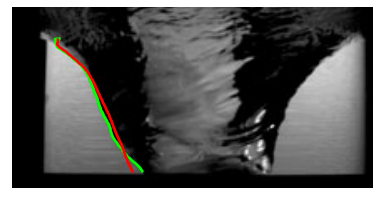

$t=T / 4$

(d)

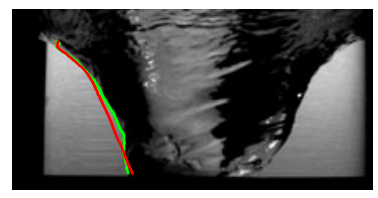

$t=3 T / 4$
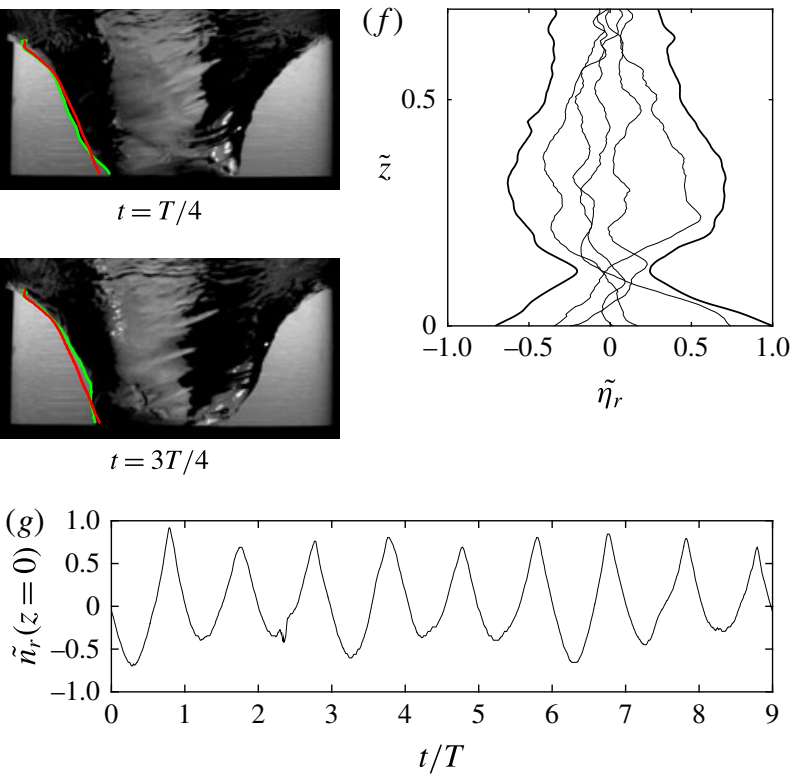

FIgURE 19. (Colour online) Experimental evidence for a secondary resonance in an experiment with rotating bottom. Polygonal state $m=3, n_{g}=0$ and $n_{c}=1$ obtained for $a=0.7$ and bottom plate rotation rate $f_{b}=4.41 \mathrm{~Hz}$. $(a-e)$ Meridional cuts during a period $T=0.27 \mathrm{~s}$ where the green curve (respectively red) corresponds to the instantaneous free surface position (respectively the free-surface position averaged over 23 periods). $(f)$ Normalized radial displacement $\tilde{\eta}_{r}$ along the free surface as a function of $\tilde{z}=z / \zeta$. $\tilde{\eta}_{r}$ is defined as the difference between instantaneous and mean free-surface positions normalized by the maximum value. Thin lines depict instantaneous values corresponding to $(a-e)$, thick lines show the envelope obtained by averaging over 23 periods. ( $g$ ) Normalized radial displacement of the contact line on the bottom plate as function of time.

interactions involving negative energy waves (Cairns 1979). In both theory the key ingredients consist of two surface waves radially separated by a critical radius.

\section{Secondary resonance: experimental evidence}

As we have discussed above, the main resonance $\left(n_{g}, n_{c}\right)=(0,0)$ is typically found to have the largest growth rate and would therefore be the best candidate for an experimentally realizable state. Thus, the system would, at least qualitatively, be well described by the simple interacting waves system introduced in Tophøj et al. (2013). However, it was noted in $\S 3.3$ that the scenario corresponding to the Tophøj model does not capture all the instabilities of the system. For instance, figure 7 shows that the secondary resonance $(0,1)$ is important for a deep-water example aspect ratio $a=$ 0.7 and an appropriate range of $\xi / R$. This result indicates that secondary resonances may be relevant experimentally, and in the following we shall show evidence that this is indeed the case.

We used an experimental set-up consisting of a cylindrical tank of radius $R=14.5 \mathrm{~cm}$ whose bottom can rotate at a controlled frequency. A more detailed description of this experimental set-up can be found in Bach et al. (2014). In figure 19, we show experimental results for the case $a=0.7$ which is larger than 
the aspect ratios previously reported for such a large cylinder radius (Jansson et al. 2006; Bach et al. 2014). As will be evidenced in the following, figure 19 corresponds to a secondary resonance $(0,1)$ for a triangular structure, i.e. $m=3$. To reach this state shown in figure 19 with a dry central core, we slowly increase the frequency $f_{b}$ of the bottom plate up to a value $f_{b}=4.41 \mathrm{~Hz}$, with fixed cylinder wall and fixed volume (corresponding to $a=0.7$ ). Other procedures can lead to different states as emphasized in Bach et al. (2014) and, indeed, proceeding e.g. by decreasing the frequency from a large initial value, leads to a 'wet' state, where no part of the free surface touches the bottom plate.

In the meridional view in figure $19(a-e)$ we see that the shape of the free surface nearly repeats after a period $T=0.27 \mathrm{~s}$ corresponding to the rotation of the triangle from one corner to the next, and we recognize, at least qualitatively, the concave free-surface shapes given by $(2.4 c)$ and figure 1. In figure 19, beyond the observed $T$-periodic signal, a $3 T$-periodic signature of the radial displacement of the contact line is observed as a slightly larger amplitude of the signal every three periods. This $3 T$-periodic response of the signal, induced by a slight asymmetry of the free-surface pattern, is associated with the triangular shape of the pattern in the azimuthal direction observed in this case.

The data corresponding to figure $19(a-e)$ allow us to track the free-surface evolution (green) and compare it to the averaged free-surface position (red). The flow is turbulent, but the free-surface structure is nearly periodic as shown in figure $19(\mathrm{~g})$, which displays the temporal evolution of the radius of the contact line on the bottom plate $(r=\xi$ in the symmetrical case). The differences between instantaneous free-surface positions and mean value are shown in figure $19(f)$ where the envelope, shown by the thick lines, is obtained by averaging over several periods. Interestingly, we find a node in the radial structure in contrast with the classical polygonal shapes obtained in e.g. Vatistas (1990) or Jansson et al. (2006). The state can therefore be related to a secondary resonance $\left(n_{g}, n_{c}\right)=(0,1)$ which is associated with the fundamental gravity wave $G_{0}$ and the first centrifugal wave harmonic $C_{1}$, which has a node in the nearly vertical part of the free surface, i.e. close to the contact line on the bottom plate. A comparison between the experimental state and the resonance $\left(n_{g}, n_{c}\right)=(0,1)$, obtained for $m=3$ in the global stability and shown in figure 7 , is made in figure 20 . Figure $20(b, d)$ displays a free surface whose structure is close to the experimental observation described above, with in particular the node corresponding to the centrifugal wave $C_{1}$ which can be clearly identified. In addition, comparison between figure $20(c, d)$ shows that this node is approximatively located at the same position $z / \zeta \approx 0.12$ in the experiment and in the theory.

For a more quantitative comparison, the frequency of the triangular pattern found experimentally can be extracted from figure $19(f)$ and compared to the frequency of the resonance found by the global stability analysis. This is done in table 1 where the theoretical frequencies for the main resonances are also compared with recent experimental results of Bach et al. (2014). It can be seen that for both the secondary resonance $\left(n_{g}, n_{c}\right)=(0,1)$ and the main resonances, good agreement is found between the linear stability predictions and the experiments. In particular, this comparison supports the results by Tophøj et al. (2013) that the main resonance is closely related to the appearance of the rotating polygons, and that the new observed pattern indeed corresponds to a secondary resonance. The fact that secondary resonances have not been seen in earlier experiments may be associated with their narrow unstable ranges and small growth rates found in the theory (see, e.g. figure 3). 
(a)

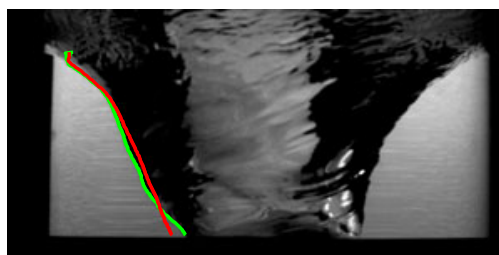

(c)

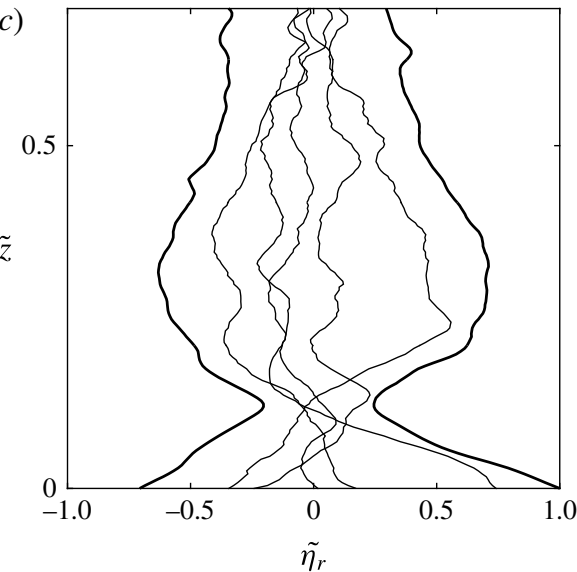

(b)

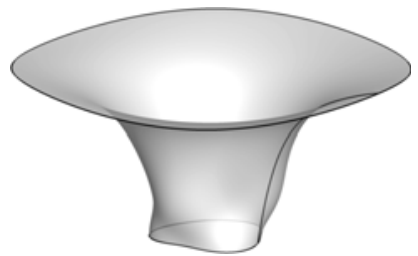

$(d)$

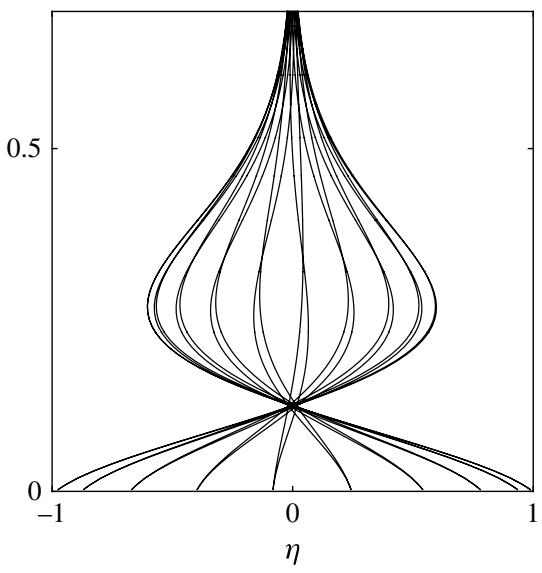

FIgURE 20. (Colour online) Surface shape and structure of a secondary resonance from experiment $(a, c)$ and theory $(b, d)$. Panels $(a)$ and $(c)$ are identical to figure $19(b, f)$ respectively. Panels $(b, d)$ correspond to the secondary resonance $(0,1)$ from global stability theory with $m=3, a=0.7$ and $\xi / R=0.28$; $(b)$ shows a $3-\mathrm{D}$ free-surface reconstruction; (d) depicts the structure of the mode by displaying $\operatorname{Re}\left(\eta \mathrm{e}^{-\mathrm{i}(m \theta-\omega t)}\right)$ for various values of $\theta$ and a given value of $t$. The reconstructions are obtained from the pressure field and $(2.8 d)$ using an arbitrary amplitude.

$\begin{array}{ccccc}m & \left(n_{g}, n_{c}\right) & a & \text { Experiment } & \text { Theory } \\ \mathbf{3} & (\mathbf{0}, \mathbf{1}) & \mathbf{0 . 7} & \mathbf{2 . 8} & \mathbf{3 . 4 7} \\ 2 & (0,0) & 0.34 & \sim 1.3-1.6 & 2.06 \\ 3 & (0,0) & 0.34 & \sim 2.6-2.9 & 3.26 \\ 4 & (0,0) & 0.34 & \sim 4.4-4.6 & 4.69\end{array}$

TABLE 1. Comparison between experiment and theory for the frequencies of a set of polygonal states. The values given are the normalized frequencies $(\omega \sqrt{R / g})$. For the theoretical values, computed by global linear analysis, we use the real part $\omega_{r}$, taken at the point of maximal growth rate in the instability range. Experimental value for the bold line corresponds to results shown in figure 19, other lines correspond to experimental results from Bach et al. (2014) evaluated from their Figure 15. Note that the experimental values are obtained from the fully developed polygons, which might differ from those obtained from linear analysis. Note also that the instability locus cannot be compared directly between potential theory and experiments as the associated controlled parameters $(\xi / R$ and $f_{b}$ respectively) are not related $a$ priori and an additional model should be introduced (see Tophøj et al. 2013; Fabre \& Mougel 2014). 


\section{Conclusion and perspectives}

In this paper, a detailed analysis of the linear stability of the potential vortex with a free surface is presented. The global stability analysis shows that a potential vortex with a free surface may become unstable in particular ranges of the parameter $\xi / R$ for $m \geqslant 2$ due to resonance between two surface wave families: gravity waves propagating where the free surface is nearly flat (close to the cylinder wall) and centrifugal waves propagating on the more strongly deformed free surface near the dry central vortex core. Beyond a full description of the wave families and their interaction, we provide new light on the instability mechanism by a WKBJ analysis conducted in the shallow-water limit. This analysis allows us to interpret the instability mechanism of the obtained instabilities in terms of wave over-reflection.

In the global stability map, the strongest unstable modes are often associated with what we have called the main resonances between gravity and centrifugal waves. They correspond to the resonances found in the Tophøj model (Tophøj et al. 2013) and argued to be closely related to the emergence of rotating polygons in the rotating bottom experiment. But in the present analysis we generalize this instability mechanism, i.e. a resonance between two wave types, by experimentally reporting the occurrence of a new polygonal pattern (triangle, i.e. $m=3$ ) with a more complex radial structure which presents an additional node on the free surface. This pattern appears to correspond to the resonance between a gravity wave with the simplest structure and a more complex centrifugal wave in the stability analysis.

In the same kind of experimental set-up as used in the present study, states with broken rotation symmetry of the free surface can also be found in wet cases for which the liquid covers the entire plate (Jansson et al. 2006). The flow in those cases cannot be modelled by a simple potential flow and the azimuthal velocity is believed to approach a Rankine like profile (Bergmann et al. 2011) containing a central core in solid body rotation. This model has been first considered by Vatistas, Wang \& Lin (1994) and the extension of the Tophøj model to the case of a Rankine vortex is provided in Fabre \& Mougel (2014) and gives an interpretation of the mechanisms of the sloshing phenomena (Iga et al. 2014) in terms of wave interactions. However, the central core in solid body rotation corresponds to a Newton's bucket flow which has been analysed in Mougel, Fabre \& Lacaze (2015), where it was shown that inertial waves and Rossby waves play an important role. These waves are not captured by the extended Tophøj model from Fabre \& Mougel (2014) and might lead to additional resonances in the Rankine vortex case. The global stability of a Rankine vortex (or a smoothed Rankine vortex) with a free surface therefore deserves to be studied more carefully to complement preliminary results presented in Mougel et al. (2014). In addition, more realistic base flows corresponding to the rotating bottom experiment have been computed numerically by Kahouadji \& Witkowski (2014) in wet cases and global stability of those flows remains to be investigated in strong free-surface deformation regimes.

The present linear stability results predict instability in thin regions of the parameter space $(a, \xi / R)$. This is in contrast with the experimental observations where polygonal patterns have been observed over large ranges of parameters (see Tophøj et al. 2013, figure 4). However, evidence of hysteresis phenomenon has been found experimentally by Tasaka \& Iima (2009) when upward and downward variations of the bottom frequency are performed. This feature is presumably associated with subcritical bifurcations, and may explain the discrepancy between linear stability thresholds and 
experimental observations. In further investigations, considering weakly nonlinear analysis of the present potential vortex could be an attractive way to investigate the above discrepancy in more depth.

To conclude, we stress the strong connection between the present study and geophysical investigations e.g. by Schecter \& Montgomery (2004, 2006). In these studies devoted to understanding geophysical vortices, such as hurricanes or the polar vortex, features such as a critical radius and interacting wave families are also reported (see e.g. the works by Schecter \& Montgomery (2004) and Billant \& Le Dizès (2009)). In addition, broken axial rotation symmetry occurs spectacularly for geophysical vortices such as the polygonal eye walls of hurricanes observed by Lewis \& Hawkins (1982). Simplified laboratory experiments, such as the rotating bottom experiment, may therefore give valuable insights into complex geophysical flows with rotation combined with a free surface or stratification.

\section{Acknowledgement}

J.M. thanks E. Linnartz for his help regarding post-treatment of experimental results.

\section{Appendix A. Global stability results for $m=0$ and $m=1$}

For $m=0$ and $m=1$, instabilities have not been observed from the global stability approach in the investigated range of parameters. Additional information corresponding to these cases are given in this appendix. In the case $a=0.3$, results for the frequencies as a function of $\xi / R$ are reported in figure 21 and the corresponding growth rate is zero. The obtained mode branches therefore correspond to neutral waves. Compared to the case $m \geqslant 2$ whose typical result can be seen in figure 12, the two wave families, i.e. gravity and centrifugal, are not easily discernible in figure $21(a, b)$.

The axisymmetric case $m=0$ is similar to sloshing modes in a U-shape tube in which the free-surface motion at one end of the tube is simply linked to the other by conservation of the volume. In this sense, pure gravity wave and pure centrifugal waves are more unlikely to appear for $m=0$ as confirmed from the mode structures shown in figure 22 where it can be seen that free-surface deformations are neither located only close to $r=\xi$ nor only close to $r=R$ contrary to the case $m \geqslant 2$ (see figures 5 and 6). For $m \geqslant 2$, we have found that the area of coexistence of these two wave families is closely related with the presence of a critical radius in the fluid domain (see figure 12). The absence of critical radius in the range $[\xi, R]$ for $m=0$ (as $\left.r_{c}=\sqrt{m \Gamma /\left(2 \pi \omega_{r}\right)}\right)$ can therefore explain why the two wave families are not obtained and hence no crossing leading to instability occurs.

In the case $m=1$, mode structures shown in figure 23 resemble the first centrifugal wave (figure 23a) and the two first gravity waves (figure $23 b, c$ respectively), but no mode crossing leading to instability is found in the investigated range of parameters in spite of the fact that a critical radius may be present as shown in figure 21(b) (grey area). However, the size of this area where a critical radius is present is found to be really small compared to figure 12 for instance, and is restricted to small values of $\xi / R$. This may explain why instabilities are not obtained here, but we cannot exclude instabilities for $m=1$ may be found for small values of $\xi / R$. 

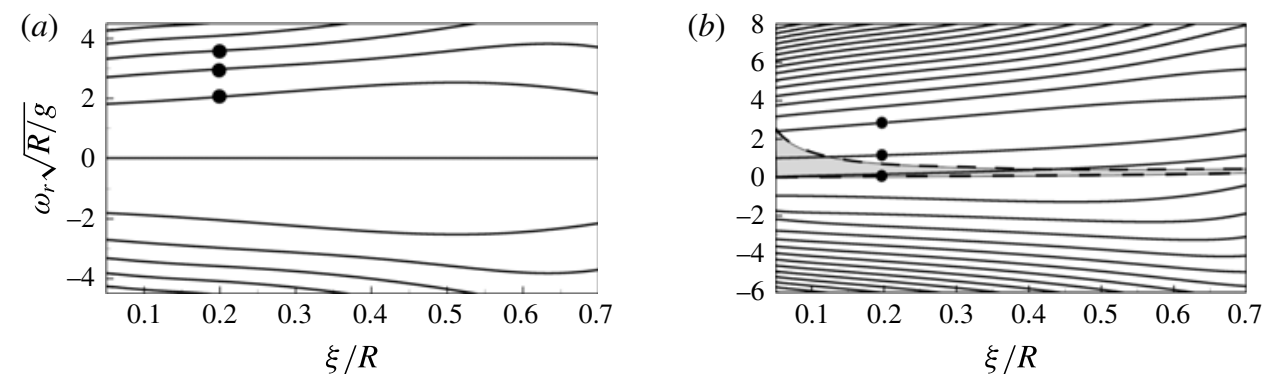

FIgURE 21. Normalized frequencies $\omega_{r} \sqrt{R / g}$ as a function of $\xi / R$ for $a=0.3$ and $m=0(a)$ and $m=1(b)$. The shaded area between the dashed lines shows the region where a critical radius is present in the fluid domain, i.e. $\xi<r_{c}<R$. Upper dashed line corresponds to $\omega_{r}=m \Gamma /\left(2 \pi \xi^{2}\right)$ (critical radius at $r=\xi$ ), lower dashed line corresponds to $\omega_{r}=m \Gamma /\left(2 \pi R^{2}\right)$ (critical radius at $r=R$ ). Black dots correspond to mode structures shown in figures 22 and 23.
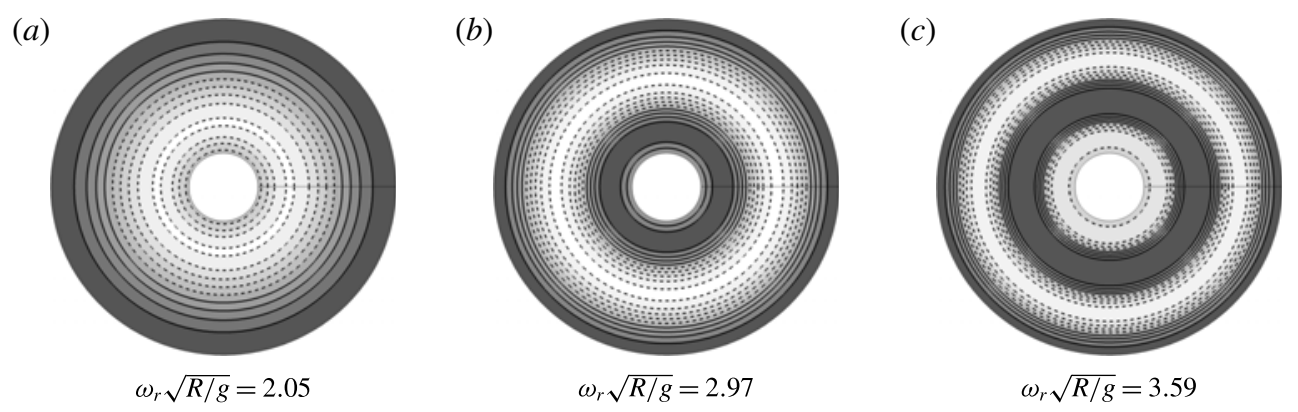

FIGURE 22. Mode structures for $a=0.3, \xi / R=0.2$ and $m=0$.
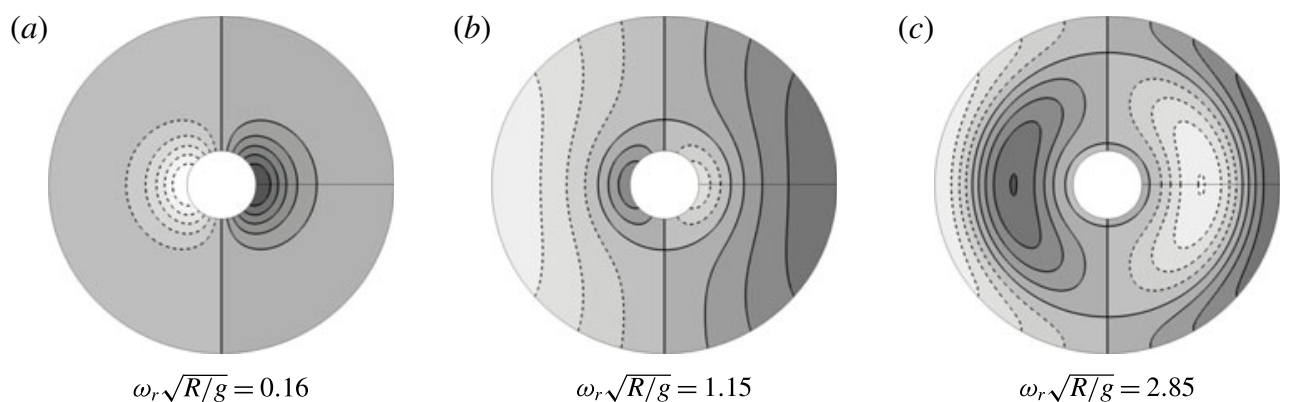

FIGURE 23. Mode structures for $a=0.3, \xi / R=0.2$ and $m=1$.

\section{Appendix B. Details on viscous potential results}

Details on the viscous and potential stability analysis are provided in this appendix. Viscosity is introduced here in order to filter out the numerous weak resonances appearing for small values of $\xi / R$ in the inviscid case (see figure 9 for instance). This method therefore allows us to construct more comprehensible stability maps where only the strongest resonances are shown (figures 2 and 3). 


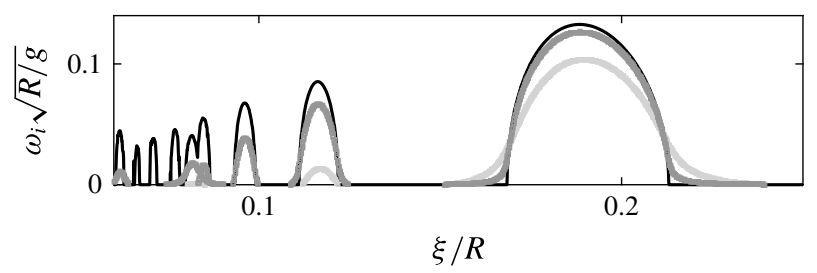

FIGURE 24. Influence of $C$ on the normalized growth rates from global stability as a function of $\xi / R$ for $a=0.3$ and $m=2$. Inviscid i.e. $C=0$ (black line); $C=1 \times 10^{-4}$ (dark grey); $C=5 \times 10^{-4}$ (light grey).

Following Funada \& Joseph (2001), viscous effects can be introduced in the velocity potential formulation given by the set of (2.8) via the dynamic boundary condition on the free surface $(2.8 d)$ which can be re-written as

$$
p-2 v \frac{\partial^{2} \phi}{\partial n^{2}}=g \eta,
$$

with $v$ the kinematic viscosity of the fluid.

This leads us to introduce the normalized viscosity,

$$
C=\frac{v}{R \sqrt{g R}}
$$

whose inverse may be regarded as a Reynolds number based on gravity.

Figure 24 displays the instability growth rate of both main and secondary instabilities for three values of $C$. For $C=0$ we obtain the purely inviscid results detailed in the present paper (except for figures 2 and 3). In this case, either neutral waves (i.e. $\left.\omega_{i}=0\right)$ or couples of complex conjugate solutions $\left(\omega_{r} \pm i \omega_{i}\right)$ are found. In figure 24, this means that the black line corresponding to inviscid results has an exactly symmetric counterpart for negative growth rate. For non-zero values of $C$, the dissipation introduced breaks this symmetry. As illustrated in figure 24 for $C=1 \times 10^{-4}$ and $C=5 \times 10^{-4}$, the introduced viscosity reduces the maximal growth rate of each resonance. More precisely, this effect is more important for the weakest resonances which may disappear for high enough values of $C$. In addition, some unstable bands are found to be larger for non-zero values of $C$ e.g. in figure 24 for the main resonance.

Finally, results shown in figure 24 reveal that for $C=1 \times 10^{-4}$, viscosity allows us to filter out the smallest resonances without having a strong effect on the location and width of the largest unstable bands. This value of $C$ has therefore been considered to compute the instability maps displayed in figures 2 and 3 . All the other results presented in this paper are purely inviscid. Note that the full description of the role played by viscosity in the present system falls outside the scope of this study, and may include non-intuitive phenomena as pointed out by Cairns (1979) in a simpler configuration.

\section{Appendix C. WKBJ large $m$ analysis in the shallow-water limit}

In this appendix, we provide details regarding the WKBJ approach to obtain approximate dispersion relations of the shallow-water equation

$$
\frac{\mathrm{d}^{2} \hat{\phi}}{\mathrm{d} r^{2}}+\left(\frac{1}{r}+\frac{h_{0}^{\prime}}{h_{0}}\right) \frac{\mathrm{d} \hat{\phi}}{\mathrm{d} r}-m^{2} \Lambda(r) \hat{\phi}=0,
$$


with

$$
h_{0}(r)=\frac{1}{2 g}\left(\frac{\Gamma}{2 \pi R}\right)^{2}\left(\frac{R^{2}}{\xi^{2}}-\frac{R^{2}}{r^{2}}\right)
$$

and

$$
\Lambda(r)=\frac{1}{r^{2}}-\frac{1}{g h_{0}}\left(\frac{\omega}{m}-\frac{\Gamma}{2 \pi r^{2}}\right)^{2},
$$

in the large $m$ limit.

Looking for a solution of the form

$$
\hat{\phi}(r) \sim \mathrm{e}^{m\left(\psi_{0}+\psi_{1} / m+\cdots\right)},
$$

equation $(\mathrm{C} 1)$ can then be expanded in orders of $m$. The two first orders $\left(m^{2}\right.$ and $\left.m\right)$ give the equations

$$
\begin{gathered}
\left(\frac{\mathrm{d} \psi_{0}}{\mathrm{~d} r}\right)^{2}-\Lambda(r)=0, \\
\frac{\mathrm{d}^{2} \psi_{0}}{\mathrm{~d} r^{2}}+2 \frac{\mathrm{d} \psi_{0}}{\mathrm{~d} r} \frac{\mathrm{d} \psi_{1}}{\mathrm{~d} r}+\left(\frac{1}{r}+\frac{h_{0}^{\prime}}{h_{0}}\right) \frac{\mathrm{d} \psi_{0}}{\mathrm{~d} r}=0,
\end{gathered}
$$

whose solutions lead to the rapidly varying phase

$$
\begin{gathered}
m \psi_{0}= \pm m \int_{r} \sqrt{\Lambda(r)} \mathrm{d} r \quad \text { if } \Lambda(r)>0, \\
m \psi_{0}= \pm \mathrm{i} m \int_{r} \sqrt{-\Lambda(r)} \mathrm{d} r \quad \text { if } \Lambda(r)<0,
\end{gathered}
$$

and the slowly varying amplitude

$$
\mathrm{e}^{\psi_{1}}=|\Lambda|^{-(1 / 4)} r^{-(1 / 2)} h_{0}^{-(1 / 2)}
$$

Equations (C4), (C 7) and (C 8) provide two leading-order solutions for (C 1) which will be referred to as the WKBJ solutions. From $(\mathrm{C} 7)$ and $(\mathrm{C} 8)$ it can be seen that these solutions are locally evanescent (if $\Lambda(r)>0$ ) or oscillatory (if $\Lambda(r)<0$ ) in the radial direction. In addition, these WKBJ solutions break down at radial locations corresponding to $\Lambda(r)=0$ (the so-called turning points) or $h_{0}(r)=0(r=\xi)$. In both cases, one has to look at the local solution of the shallow-water equation, (C1), around these special points and match these solutions to the WBKJ solutions. This allows us to obtain an approximate dispersion relation.

We now restrict our attention to configuration III, that is two oscillatory regions separated by an evanescent region which includes a critical radius (see figure $15 d$ ). There are therefore two turning points $r_{t 1}$ and $r_{t 2}$ nearby which the WKBJ approximation breaks down. The evanescent region and the outer oscillatory region will be treated using WKBJ approximation while the inner oscillatory region, whose size is very small in the large $m$ limit, will be included in the treatment of the equation close to $\xi$. WKBJ solutions read

$$
\hat{\phi}=\frac{1}{|\Lambda|^{1 / 4} r^{1 / 2} h_{0}^{1 / 2}}\left[A \exp \left(\mathrm{i} m \int_{r_{t 2}}^{r} \sqrt{-\Lambda}\right)+B \exp \left(-\mathrm{i} m \int_{r_{t 2}}^{r} \sqrt{-\Lambda}\right)\right],
$$


for $r_{t 2}<r<R$, and

$$
\hat{\phi}=\frac{1}{|\Lambda|^{1 / 4} r^{1 / 2} h_{0}^{1 / 2}}\left[C \exp \left(m \int_{r}^{r_{t 2}} \sqrt{\Lambda}\right)+D \exp \left(-m \int_{r}^{r_{t 2}} \sqrt{\Lambda}\right)\right],
$$

for $r_{t 1}<r<r_{t 2}$.

At $r=R$, the no-penetration boundary condition $\mathrm{d} \hat{\phi} / \mathrm{d} r=0$ leads, at leading order, to

$$
\frac{B}{A}=\mathrm{e}^{2 \mathrm{i} m W_{2 R}(\omega)},
$$

where

$$
W_{2 R}(\omega)=\int_{r_{t 2}}^{R} \sqrt{-\Lambda(r)} \mathrm{d} r .
$$

We now focus on the behaviour of the solutions close to the turning point $r_{t 2}$. Introducing the rescaled variable $\tilde{r}=\left(r-r_{t 2}\right)\left[-\Lambda^{\prime}\left(r_{t 2}\right) m^{2}\right]^{1 / 3}$ with $\Lambda^{\prime}=\mathrm{d} \Lambda / \mathrm{d} r$, equation (C 1) leads, at leading order, to

$$
\frac{\mathrm{d}^{2} \hat{\phi}}{\mathrm{d} \tilde{r}^{2}}+\tilde{r} \hat{\phi}=0
$$

Solutions of (C 14) read

$$
\hat{\phi}=A_{1} \mathrm{~A}_{i}(-\tilde{r})+A_{2} \mathrm{~B}_{i}(-\tilde{r})
$$

with $\mathrm{A}_{i}$ and $\mathrm{B}_{i}$ Airy functions and $A_{1}, A_{2}$ two constants. Behaviour of Airy functions when $\tilde{r} \rightarrow \infty$ and $\tilde{r} \rightarrow-\infty$ allows us respectively to match with WKBJ solutions in the oscillatory zone $((\mathrm{C} 10)$ for $\tilde{r} \rightarrow \infty)$ and evanescent zone $((\mathrm{C} 11)$ for $\tilde{r} \rightarrow-\infty)$. This leads to the conditions

$$
\frac{B}{A}=\mathrm{i} \frac{1-\mathrm{i} A_{2} / A_{1}}{1+\mathrm{i} A_{2} / A_{1}} \quad \text { and } \quad \frac{D}{C}=\frac{A_{1}}{2 A_{2}} .
$$

This analysis around a turning point is classical and we refer the interested reader to Bender \& Orszag (1999) (for instance) for additional details. The combination of the two obtained conditions, along with (C 12) finally lead to the relation

$$
\frac{D}{C}=\frac{\mathrm{i}}{2}\left(\frac{1-\mathrm{i} B / A}{1+\mathrm{i} B / A}\right)=\frac{1}{2} \tan \left(m W_{2 R}(\omega)+\pi / 4\right) .
$$

The behaviour close to the contact line at $r=\xi$ is not classical due to the fact that $h_{0}$ vanishes at that radial location. Assuming that $\omega-m \Omega(\xi)=O(\sqrt{m})$, the leadingorder equation in the neighbourhood of $r=\xi$ reads

$$
\frac{\mathrm{d}^{2} \hat{\phi}}{\mathrm{d} \bar{r}^{2}}+\frac{1}{\bar{r}} \frac{\mathrm{d} \hat{\phi}}{\mathrm{d} \bar{r}}+\left[\frac{C_{1}}{\bar{r}}-C_{2}\right] \hat{\phi}=0
$$

with $\bar{r}=(r-\xi) m$ a rescaled variable, $C_{1}=m^{-1}(\omega-m \Omega(\xi))^{2} / g_{c}(\xi)$ and $C_{2}=1 / \xi^{2}$. Solutions of this equation can be obtained in the form

$$
\hat{\phi}(\bar{r})=A_{1} M(\alpha, 1,2 \bar{r} / \xi) \mathrm{e}^{-\bar{r} / \xi}+A_{2} U(\alpha, 1,2 \bar{r} / \xi) \mathrm{e}^{-\bar{r} / \xi},
$$


with $M$ and $U$ being the Kummer functions (see Abramowitz \& Stegun (1964), p. 502) and $\alpha=1 / 2\left(1-C_{1} / \sqrt{C_{2}}\right)$. From the behaviour of Kummer functions when $\bar{r} \rightarrow 0$, we must have $A_{2}=0$ to ensure finite values of the velocity potential. In the limit $\bar{r} \rightarrow \infty$, the asymptotic behaviour of the Kummer function $M$ in this limit (see Abramowitz \& Stegun (1964), p. 508) leads to solutions of (C 18) of the form

$$
\hat{\phi}(\bar{r}) \sim\left[\frac{\mathrm{e}^{\mathrm{i} \pi \alpha} 2^{-\alpha} C_{2}^{-(\alpha / 2)}}{\Gamma(1-\alpha)}\right] \bar{r}^{-\alpha} \mathrm{e}^{-\sqrt{C_{2}} \bar{r}}+\left[\frac{2^{\alpha-1} C_{2}^{(\alpha-1) / 2}}{\Gamma(\alpha)}\right] \bar{r}^{\alpha-1} \mathrm{e}^{\sqrt{C_{2}} \bar{r}},
$$

with $\boldsymbol{\Gamma}$ the gamma function. This solution should be matched with WKBJ solutions given by $(\mathrm{C} 11)$ and taken in the limit $\bar{r} \rightarrow \infty$, that is

$$
\hat{\phi}(\bar{r}) \sim C\left[\mathrm{e}^{m W_{12}(\omega)} C_{2}^{(1-2 \alpha) / 4}\right] \bar{r}^{-\alpha} \mathrm{e}^{-\sqrt{C_{2}} \bar{r}}+D\left[\mathrm{e}^{-m W_{12}(\omega)} C_{2}^{(2 \alpha-1) / 4}\right] \bar{r}^{\alpha-1} \mathrm{e}^{\sqrt{C_{2}} \bar{r}},
$$

where

$$
W_{12}(\omega)=\int_{r_{t 1}}^{r_{t 2}} \sqrt{\Lambda(r)} \mathrm{d} r
$$

It should be noted that the same radial evolution is obtained in (C 20) and (C 21), which allows for a proper matching leading to the condition

$$
\frac{\boldsymbol{\Gamma}(1-\alpha)}{\boldsymbol{\Gamma}(\alpha)}=\left[2^{1-2 \alpha} \mathrm{e}^{\mathrm{i} \pi \alpha} \mathrm{e}^{-2 m W_{12}(\omega)}\right] \frac{D}{C} .
$$

Note that the inner wavy region vanishes in the large $m$ limit as $r_{t 1}$ tends to $\xi$, this region is described within the range of validity of solution (C 19), and this is the reason why we directly match solutions near $r=\xi$ to WKBJ solutions in the evanescent region.

WKBJ solutions in the evanescent and oscillatory area have been matched to the obtained solutions close to $r=\xi$ and $r=r_{t 2}$, and the no-penetration boundary condition has been applied at $r=R$. This procedure leads to (C 17) and (C 23), whose combination give the approximate dispersion relation

$$
\left[\frac{\boldsymbol{\Gamma}(1-\alpha)}{\boldsymbol{\Gamma}(\alpha)}\right]\left[\frac{1}{\tan \left(m W_{2 R}(\omega)+\pi / 4\right)}\right]=2^{-2 \alpha} \mathrm{e}^{\mathrm{i} \pi \alpha} \mathrm{e}^{-2 m W_{12}(\omega)} .
$$

It should be noted that the left-hand side of this dispersion relation vanishes at the singularities of the gamma function or those of the tangent function. These singularities respectively correspond to $\alpha=-n_{c}$ and $m W_{2 R}(\omega)+\pi / 4=\pi / 2+n_{g} \pi$ with $n_{c}$ and $n_{g}$ two integers, and those discretizations lead to the following conditions which may be regarded as dispersion relations

$$
\begin{gathered}
(\omega-m \Omega(\xi))^{2}=g_{c}(\xi) \frac{m}{\xi}\left(1+2 n_{c}\right), \\
m W_{2 R}(\omega)=\pi / 4+n_{g} \pi .
\end{gathered}
$$

Dispersion relation (C 25) corresponds to the so-called centrifugal waves evolving in the inner oscillatory region, and can be interpreted as edge waves in this shallow limit (see Ursell 1952; Mougel et al. 2015). Dispersion relation (C 26) corresponds to gravity waves that propagate in the outer oscillatory region. 
In addition, the right-hand side of (C24) goes to zero in the large $m$ limit and the complete dispersion relation given by (C 24) can therefore be interpreted as two uncoupled dispersion relations (corresponding to pure centrifugal and pure gravity waves) which equals a small coupling term. This is reminiscent of the dispersion relation describing the main resonances in the Tophøj model (Tophøj et al. 2013), but the present formalism also includes secondary resonances.

The aim is now to explore (C24) in order to find more explicit solutions for the frequencies. The right-hand side of equation (C24) is small in the large $m$ limit, which makes the relation possible only near the singularities of the gamma and tangent function discussed above. From the behaviour of the gamma function around its singularities (zero and negative integer values), which are described by the relation

$$
\boldsymbol{\Gamma}(\epsilon-n)=\frac{(-1)^{n}}{\Gamma(n+1) \epsilon} \text { for } \epsilon \rightarrow 0 \text { and with } n \in \mathbb{N},
$$

and using in addition the relation $\Gamma(n+1)=n$ !, the dispersion relation $(\mathrm{C} 24)$ can be written at leading order in the form

$$
(\omega-m \Omega(\xi))^{2}=g_{c}(\xi) \frac{m}{\xi}\left(1+2 n_{c}\right)-g_{c}(\xi) \frac{m}{\xi} \frac{2^{2 n_{c}+1}}{\left(n_{c} !\right)^{2}} \tan \left(m W_{2 R}(\omega)+\pi / 4\right) \mathrm{e}^{-2 m W_{12}(\omega)} .
$$

Equation (C 28) can be seen as a generalization of dispersion relation (C 25) for the centrifugal waves. Consequently the integer $n$ has been noted $n_{c}$ in equation (C28). Note however that (C28) now includes coupling with gravity waves which are hidden in the term with the tangent function. When solving (C 28), only the solution corresponding to a centrifugal wave slower than the base flow $(\omega-m \Omega(\xi)<0)$ is relevant as the other solution falls outside of the range of frequency corresponding to area III in which the present analysis is valid. We obtain at leading order

$$
\begin{aligned}
\omega= & m \Omega(\xi)-\sqrt{g_{c}(\xi) \frac{m}{\xi}} \sqrt{1+2 n_{c}} \\
& +\sqrt{g_{c}(\xi) \frac{m}{\xi}} \frac{2^{2 n_{c}}}{\sqrt{1+2 n_{c}}\left(n_{c} !\right)^{2}} \tan \left(m W_{2 R}(\omega)+\pi / 4\right) \mathrm{e}^{-2 m W_{12}(\omega)} .
\end{aligned}
$$

In the particular case $n_{c}=0$, (C 29) finally leads to (4.8) whose solutions correspond to the first centrifugal wave with possible interaction with gravity waves.

We now look at the way gravity waves are reflected back when they encounter the evanescent region. The reflection coefficient of a gravity wave on the potential barrier can be defined as $\mathcal{R} \equiv\left|B^{2} / A^{2}\right|$. This definition is built in analogy with the work of Billant \& Le Dizès (2009). From (C 12) the reflection coefficient reads

$$
\mathcal{R}=\left|\exp \left(\mathrm{i} 4 m W_{2 R}(\omega)\right)\right|,
$$

and is found to be larger than unity for solutions of (C 29) corresponding to unstable modes (see figure 18). The instability mechanism is therefore associated with overreflections on the evanescent area.

An interesting limit corresponds to the case of an unbounded vortex $(R \rightarrow \infty)$. In this limit $\tan \left(m W_{2 R}(\omega)+\pi / 4\right)=i$ and (C 29) therefore shows that the potential solution is always unstable in this case. This result can also be obtained considering 
a radiation condition for the waves in the outer oscillatory zone, i.e. $B=0$. This latter instability therefore corresponds to a radiative instability where the vortex becomes unstable due to emission of gravity waves towards infinity. Interestingly, the unbounded shallow-water vortex studied by Ford (1994) for more general azimuthal velocity profiles are also found to be always unstable due to gravity wave radiation. In addition, a more detailed discussion between wave resonances in the bounded case and radiative instability in the unbounded case can be found in Le Dizès \& Riedinger (2010) for a stratified vortex.

\section{REFERENCES}

Abramowitz, M. \& Stegun, I. A. 1964 Handbook of Mathematical Functions: With Formulas, Graphs, and Mathematical Tables, vol. 55. Courier Corporation.

ACHESON, D. J. 1976 On over-reflexion. J. Fluid Mech. 77 (03), 433-472.

Bach, B., Linnartz, E. C., Vested, M. H., Andersen, A. \& Bohr, T. 2014 From Newton's bucket to rotating polygons: experiments on surface instabilities in swirling flows. J. Fluid Mech. 759, 386-403.

Bender, C. M. \& Orszag, S. A. 1999 Advanced Mathematical Methods for Scientists and Engineers I. Springer.

Bergmann, R., Tophøj, L., Homan, T. A. M., Hersen, P., Andersen, A. \& Bohr, T. 2011 Polygon formation and surface flow on a rotating fluid surface. J. Fluid Mech. 679, 415-431.

Billant, P. \& LE DizÈs, S. 2009 Waves on a columnar vortex in a strongly stratified fluid. Phys. Fluids 21 (10), 106602.

CAIRns, R. A. 1979 The role of negative energy waves in some instabilities of parallel flows. J. Fluid Mech. 92, 1-14.

Fabre, D. \& Mougel, J. 2014 Generation of three-dimensional patterns through wave interaction in a model of free surface swirling flow. Fluid Dyn. Res. 46 (6), 061415.

FORD, R. 1994 The instability of an axisymmetric vortex with monotonic potential vorticity in rotating shallow water. J. Fluid Mech. 280, 303-334.

Funada, T. \& JOSEPH, D. D. 2001 Viscous potential flow analysis of Kelvin-Helmholtz instability in a channel. J. Fluid Mech. 445, 263-283.

HeCHT, F. 2012 New development in freefem++. J. Numer. Maths 20 (3-4), 251-265.

Iga, K., Yokota, S., Watanabe, S., Ikeda, T., Ninno, H. \& Misawa, N. 2014 Various phenomena on a water vortex in a cylindrical tank over a rotating bottom. Fluid Dyn. Res. 46 (3), 031409.

IIMA, M. \& TASAKA, Y. 2016 Dynamics of flow structures and surface shapes in the surface switching of rotating fluid. J. Fluid Mech. 789, 402-424.

Jansson, T. R. N., Haspang, M. P., Jensen, K. H., Hersen, P. \& Bohr, T. 2006 Polygons on a rotating fluid surface. Phys. Rev. Lett. 96, 174502.

Kahouadji, L. \& Witkowski, L. M. 2014 Free surface due to a flow driven by a rotating disk inside a vertical cylindrical tank: axisymmetric configuration. Phys. Fluids 26 (7), 072105.

LE Dizès, S. \& LACAZE, L. 2005 An asymptotic description of vortex Kelvin modes. J. Fluid Mech. 542, 69-96.

LE DizÈs, S. \& Riedinger, X. 2010 The strato-rotational instability of Taylor-Couette and Keplerian flows. J. Fluid Mech. 660, 147-161.

Lewis, B. M. \& Hawkins, H. F. 1982 Polygonal eye walls and rainbands in hurricanes. Bull. Am. Meteorol. Soc. 63 (11), 1294-1301.

LINDZEN, R. S. \& BARKER, J. W. 1985 Instability and wave over-reflection in stably stratified shear flow. J. Fluid Mech. 151, 189-217.

Mougel, J., FAbre, D. \& LACAZE, L. 2014 Waves and instabilities in rotating free surface flows. Mech. Ind. 15, 107-112.

Mougel, J., Fabre, D. \& Lacaze, L. 2015 Waves in Newton's bucket. J. Fluid Mech. 783, 211-250. 
PARK, J. \& Billant, P. 2013 The stably stratified Taylor-Couette flow is always unstable except for solid-body rotation. J. Fluid Mech. 725, 262-280.

Schecter, D. A. \& Montgomery, M. T. 2004 Damping and pumping of a vortex Rossby wave in a monotonic cyclone: critical layer stirring versus inertia-buoyancy wave emission. Phys. Fluids 16 (5), 1334-1348.

Schecter, D. A. \& Montgomery, M. T. 2006 Conditions that inhibit the spontaneous radiation of spiral inertia-gravity waves from an intense mesoscale cyclone. J. Atmos. Sci. 63 (2), 435-456.

Suzuki, T., Ima, M. \& Hayase, Y. 2006 Surface switching of rotating fluid in a cylinder. Phys. Fluids 18 (10), 101701.

TAKehiro, S. \& HAYASHI, Y. 1992 Over-reflection and shear instability in a shallow-water model. J. Fluid Mech. 236, 259-279.

TASAKA, Y. \& IIMA, M. 2009 Flow transitions in the surface switching of rotating fluid. J. Fluid Mech. 636, 475-484.

Tорнøј, L. \& Boнr, T. 2013 Stationary ideal flow on a free surface of a given shape. J. Fluid Mech. 721, 28-45.

TophøJ, L., Mougel, J., Bohr, T. \& FABre, D. 2013 Rotating polygon instability of a swirling free surface flow. Phys. Rev. Lett. 110 (19), 194502.

Ursell, F. 1952 Edge waves on a sloping beach. Proc. R. Soc. Lond. A 214 (1116), 79-97.

Vatistas, G. H. 1990 A note on liquid vortex sloshing and Kelvin's equilibria. J. Fluid Mech. 217, 241-248.

Vatistas, G. H., WANG, J. \& Lin, S. 1994 Recent findings on Kelvin's equilibria. Acta Mechanica 103, 89-102. 